\title{
KECK LASER GUIDE STAR ADAPTIVE OPTICS MONITORING OF THE M8+L7 BINARY LHS 2397aAB: FIRST DYNAMICAL MASS BENCHMARK AT THE L/T TRANSITION $*,+,, \S$
}

\author{
Trent J. Dupuy ${ }^{1}$, Michael C. LiU ${ }^{1,3}$, and Michael J. Ireland ${ }^{2}$ \\ ${ }^{1}$ Institute for Astronomy, University of Hawai ‘i, 2680 Woodlawn Drive, Honolulu, HI 96822, USA \\ 2 School of Physics, University of Sydney, NSW 2006, Australia \\ Received 2009 February 9; accepted 2009 April 21; published 2009 June 10
}

\begin{abstract}
We present Keck laser guide star adaptive optics imaging and aperture masking observations of the M8+L7 binary LHS 2397aAB. Together with archival Hubble Space Telescope, Gemini-North, and Very Large Telescope data, our observations span 11.8 years of the binary's 14.2 year orbital period. We determine a total dynamical mass of $0.146_{-0.013}^{+0.015} M_{\odot}\left(153_{-14}^{+16} M_{\text {Jup }}\right)$. Using the combined observational constraints of the total mass and individual luminosities, the Tucson (Lyon) evolutionary models give an age for the system of $1.5_{-0.6}^{+4.1} \mathrm{Gyr}\left(1.8_{-0.8}^{+8.2} \mathrm{Gyr}\right.$ ), which is consistent with its space motion based on a comparison to the Besançon Galactic structure model. We also use these models to determine the mass ratio, giving individual masses of $0.0839_{-0.0015}^{+0.0007} M_{\odot}\left(0.0848_{-0.0012}^{+0.0010} M_{\odot}\right)$ for LHS $2397 \mathrm{aA}$ and $0.061_{-0.011}^{+0.014} M_{\odot}\left(0.060_{-0.012}^{+0.008} M_{\odot}\right)$ for LHS $2397 \mathrm{aB}$. Because LHS $2397 \mathrm{aB}$ is very close to the theoretical mass limit of lithium burning, which remains untested by dynamical masses, measuring its lithium depletion would uniquely test substellar models. We estimate a spectral type of L7 \pm 1 for LHS 2397aB, making it the first $\mathrm{L} / \mathrm{T}$ transition object with a dynamical mass determination. This enables a precise estimate of its effective temperature from Tucson (Lyon) evolutionary models of $1450 \pm 40 \mathrm{~K}(1430 \pm 40 \mathrm{~K})$, which is $200 \mathrm{~K}$ higher than estimates for young late-L companions but consistent with older late-L field dwarfs, supporting the idea that the temperature of the $\mathrm{L} / \mathrm{T}$ transition is surface gravity dependent. Comparing our temperature estimate for LHS $2397 \mathrm{aB}$ to those derived from spectral synthesis modeling for similar objects reveals consistency between evolutionary and atmospheric models at the L/T transition, despite the currently limited understanding of this phase of substellar evolution. Future dynamical masses for $\mathrm{L} / \mathrm{T}$ binaries spanning a range of surface gravity, age, and mass will provide the next critical tests of substellar models at the L/T transition.
\end{abstract}

Key words: binaries: close - binaries: general - infrared: stars - stars: low-mass, brown dwarfs - techniques: high angular resolution

Online-only material: color figures

\section{INTRODUCTION}

In the field population, the warmest brown dwarfs and the lowest mass stars are in many ways quite different. Beyond the fact that brown dwarfs by definition do not sustain internal energy generation through hydrogen fusion, while very low mass stars do, the effective temperatures of these two classes of objects span a range of $\approx 1500 \mathrm{~K}$ and more than an order of magnitude in luminosity. However, they share one important attribute, which is the presence of dust clouds in their atmospheres. Below effective temperatures of $\approx 2800 \mathrm{~K}$, photospheres are cool

\footnotetext{
* Some of the data presented herein were obtained at the W.M. Keck Observatory, which is operated as a scientific partnership among the California Institute of Technology, the University of California, and the National Aeronautics and Space Administration. The Observatory was made possible by the generous financial support of the W.M. Keck Foundation.

Based partly on observations made with the NASA/ESA Hubble Space Telescope, obtained from the data archive at the Space Telescope Institute. STScI is operated by the Association of Universities for Research in Astronomy, Inc., under the NASA contract NAS 5-26555.

$\ddagger$ Based partly on observations obtained under program ID GN-2002A-Q-6 at the Gemini Observatory, which is operated by the Association of Universities for Research in Astronomy, Inc., under a cooperative agreement with the NSF on behalf of the Gemini partnership: the National Science Foundation (United States), the Science and Technology Facilities Council (United Kingdom), the National Research Council (Canada), CONICYT (Chile), the Australian Research Council (Australia), Ministério da Ciência e Tecnologia (Brazil), and SECYT (Argentina).

$\S$ Based partly on observations made with ESO Telescopes at the Paranal Observatory under program IDs 071.C-0716 and 076.C-0139.

3 Alfred P. Sloan Research Fellow.
}

enough for particulate matter to form (e.g., Tsuji et al. 1996; Jones \& Tsuji 1997), and this dust persists for objects as cool as $\approx 1300 \mathrm{~K}$ (e.g., Chabrier et al. 2000), at which temperature the dust begins to settle below the photosphere. Thus, the formation and sedimentation of dust clouds are among the key processes driving the atmospheric physics of objects in this temperature range, which also includes the warmest extrasolar planets such as transiting hot Jupiters and young giant planets (e.g., Barman 2008; Marois et al. 2008).

In addition to providing excellent laboratories for ultracool atmospheric physics, very low mass stars and brown dwarfs are also intrinsically interesting as the lowest mass products of star formation. In particular, the properties of binary systems spanning a wide range of stellar masses can provide discriminating tests of various star formation models. While the binary properties of low-mass stars (FGKM spectral types) have been extensively studied (e.g., Duquennoy \& Mayor 1991; Fischer \& Marcy 1992), such studies have only become possible within the past decade for brown dwarfs and stars at the bottom of the main sequence. In fact, such studies are generally hampered by the fact that brown dwarfs follow a mass-luminosityage relation, rather than the simpler mass-luminosity relation for main-sequence stars. For example, a late-M field dwarf may be a young brown dwarf or a star at the bottom of the main sequence. Binaries with well-determined orbits are extremely valuable as they break this mass-age degeneracy by providing dynamical mass estimates, though very few mass 
Table 1

Keck LGS AO Observations

\begin{tabular}{cccccc}
\hline \hline Date (UT) & Start Time (UT) & Airmass & Filter & FWHM (mas) & Strehl Ratio \\
\hline 2007 Apr 22 & $07: 46$ & 1.19 & $K_{S}$ & $53.8 \pm 0.2$ & $0.277 \pm 0.006$ \\
& $07: 56$ & 1.19 & $\mathrm{CH}_{4} \mathrm{~s}^{\mathrm{a}}$ & $\ldots$ & $\ldots$ \\
2008 Jan 15 & $10: 00$ & 1.47 & $K_{S}{ }^{\mathrm{a}}$ & $\ldots$ & $\ldots$ \\
& $14: 48$ & 1.21 & $K_{S}{ }^{\mathrm{a}}$ & $\ldots$ & $\ldots$ \\
2009 Jan 23 & $14: 59$ & 1.23 & $K_{S}$ & $61.0 \pm 0.8$ & $0.201 \pm 0.017$ \\
& $12: 56$ & 1.21 & $K_{S}$ & $71.2 \pm 1.1$ & $0.152 \pm 0.015$ \\
& $13: 03$ & 1.20 & $H$ & $71.9 \pm 2.7$ & $0.059 \pm 0.006$ \\
& $13: 14$ & 1.20 & $L^{\prime}$ & $93.4 \pm 0.7$ & $0.021 \pm 0.004$ \\
& $13: 33$ & 1.19 & & $0.434 \pm 0.069$ \\
\hline
\end{tabular}

Note. ${ }^{\text {a }}$ Aperture masking observations.

determinations are available to date (e.g., Zapatero Osorio et al. 2004; Dupuy et al. 2009). In fact, mass measurements from binaries typically offer much stronger constraints on substellar models than other commonly measured parameters (e.g., age and composition) given that their higher precision, hence the concept of using binaries as "mass benchmarks" (Liu et al. 2008). In addition, binary orbits provide a wealth of other information such as the true (not projected) semimajor axis and the eccentricity, both of which have different predicted distributions under different formation scenarios (e.g., Bate 2009; Fisher 2004).

One particularly striking result of the many surveys that have searched for brown dwarf binaries is the paucity of intermediate mass ratio binaries. Although such surveys have typically been sensitive to mass ratios as small as $M_{\mathrm{B}} / M_{\mathrm{A}} \approx 0.6$, the mass ratio distribution drops sharply below unity, with $>50 \%$ of binaries having estimated mass ratios of $>0.9$ (Burgasser et al. 2007). This is in stark contrast to solar-type binaries whose mass ratio distribution peaks at $\approx 0.4$ (Duquennoy \& Mayor 1991), suggesting a different formation process for these two classes of objects. Although very unequal mass (or spectral type) brown dwarf binaries are rare, they provide the strongest coevality tests of substellar theoretical models because they offer the most leverage on constraining model isochrones.

Freed et al. (2003) discovered a faint companion to LHS $2397 \mathrm{a}^{4}$ using the curvature AO system Hokupa'a at Gemini-North, revealing one of the most unequal-flux ultracool binaries known ( $\Delta K=2.77 \pm 0.10 \mathrm{mag})$. LHS 2397a (M8) is an $\mathrm{H} \alpha$ flare star with a distance measured by Monet et al. (1992) to be $14.3 \pm 0.4 \mathrm{pc}$. Freed et al. (2003) estimated the spectral type of LHS $2397 \mathrm{aB}$ from near-infrared photometry to be $\mathrm{L} 7.5 \pm 1$. Thus, LHS $2397 \mathrm{aAB}$ represents a rare pairing of two objects at the extreme ends of the effective temperature range over which dust clouds play an important role in atmospheric physics. LHS 2397aA is just cool enough that dust is likely to persist in its atmosphere, while LHS $2397 \mathrm{aB}$ has nearly cooled to the point of becoming a dust-free $\mathrm{T}$ dwarf.

Based on Keck laser guide star adaptive optics (LGS AO) imaging and aperture masking observations from our ongoing orbital monitoring program of ultracool binaries, we present here a dynamical mass for LHS 2397aAB. Combining our Keck data with archival Hubble Space Telescope (HST), Very Large Telescope (VLT), and Gemini-North Telescope images, we measure a total mass of $0.146 \pm 0.014 M_{\odot}$, with the dominant source of uncertainty being the $3.0 \%$ error in the parallax (which translates into a $9.0 \%$ error in the mass). This is the first mass

\footnotetext{
4 LHS 2397a is the star listed between LHS 2397 and LHS 2398 in the LHS Catalog (Luyten 1979).
}

measurement for a binary containing an L/T transition brown dwarf, and we use the direct mass measurement along with the measured luminosities of both components to test evolutionary and atmospheric models in this temperature regime $(\approx 1400 \mathrm{~K})$ for the first time.

\section{OBSERVATIONS}

\subsection{HST/WFPC2-PC1}

We retrieved HST archival images of LHS 2397aAB obtained with the WFPC2 Planetary Camera (PC1) on UT 1997 April 12 (GO-6345, PI Kirkpatrick). The data comprise four F814W images with exposure times of $2 \mathrm{~s}, 1000 \mathrm{~s}, 1000 \mathrm{~s}$, and $300 \mathrm{~s}$, taken in that order in a single orbit. The companion LHS 2397aB is only seen in the latter three images, and the primary star LHS 2397aA is saturated in all but the first image. We used TinyTim (Krist 1995) to generate point-spread function (PSF) models which were fit to the data in a similar fashion to our previous work (Liu et al. 2008; Dupuy et al. 2009), thus allowing us to determine the positions and fluxes of LHS 2397aAB in each image. Saturated pixels were masked in our PSF-fitting routine. The F814W flux ratio of the binary $(4.18 \pm 0.08$ mag) was determined by comparing the mean peak flux (i.e., the model PSF's normalization constant) of LHS $2397 \mathrm{aB}$ in the latter three images to the peak flux of LHS 2397aA in the first image.

Since positional information is carried almost entirely in the core of the PSF, especially for these undersampled WFPC2 images, we could not determine precise positions for LHS 2397aA in the three images in which its core was saturated. Therefore, in order to measure the binary separation and position angle (P.A.), we had to somehow tie the latter three measurements of LHS $2397 \mathrm{aB}$ to the first shallow image of LHS 2397aA. This was accomplished by fitting a first-order polynomial to the positions of LHS $2397 \mathrm{aB}$, in order to account for any telescope drift that occurred during the observations, and extrapolating this fit to the unsaturated image of LHS $2397 \mathrm{aA}$. We found a drift in the $(x, y)$ position of LHS $2397 \mathrm{aB}$ of $(0.82 \pm 0.03,0.26 \pm$ 0.01 ) pixels $\mathrm{hr}^{-1}$ over the $0.7 \mathrm{hr}$ duration of the observations. After correcting for this drift and using a pixel scale of $45.54 \pm$ 0.01 mas pixel ${ }^{-1}, 5$ we found a separation of $274 \pm 4$ mas, and P.A. of $87.3 \pm 0.8$ (Table 2 ).

To determine the uncertainties of our position and flux measurements we simulated the data using images of single stars, allowing us to assess systematic errors in our fitting procedure. We built a library of single stars from other programs

\footnotetext{
5 This is the quoted pixel scale from the WFPC2 Instrument Handbook for Cycle 13, which is consistent with other values in the literature (e.g., see the discussion by Liu et al. 2008)
} 
Table 2

Best-Fit Binary Parameters for LHS 2397aAB

\begin{tabular}{|c|c|c|c|c|c|}
\hline Epoch (UT) & Instrument & Filter & $\rho$ (mas) & P.A. $\left({ }^{\circ}\right)$ & $\Delta m(\mathrm{mag})$ \\
\hline 1997 Apr 12 & $H S T / \mathrm{WFPC} 2-\mathrm{PC} 1^{\mathrm{a}}$ & F814W & $274 \pm 4$ & $87.3 \pm 0.8$ & $4.18 \pm 0.08$ \\
\hline 2002 Feb 7 & Gemini/Hokupa' $a^{a}$ & $K^{\prime}$ & $205 \pm 8$ & $152.0 \pm 2.4$ & $3.06 \pm 0.45$ \\
\hline 2003 May 31 & VLT/NACO ${ }^{\mathrm{a}}$ & $K_{S}$ & $168 \pm 8$ & $188.6 \pm 1.2$ & $2.75 \pm 0.16$ \\
\hline 2006 Jan 12 & VLT/NACO ${ }^{\mathrm{a}}$ & $K_{S}$ & $129 \pm 5$ & $276.2 \pm 1.4$ & $2.84 \pm 0.16$ \\
\hline \multirow[t]{3}{*}{2007 Apr 22} & Keck/NIRC2 & $K_{S}$ & $119.7 \pm 2.9$ & $348.4 \pm 0.8$ & $2.94 \pm 0.10$ \\
\hline & Keck/NIRC2 (masking) & $\mathrm{CH}_{4} \mathrm{~S}$ & $112.5 \pm 2.8$ & $347.0 \pm 1.3$ & $3.37 \pm 0.24$ \\
\hline & Keck/NIRC2 (masking) ${ }^{\mathrm{a}}$ & $K_{S}$ & $115.3 \pm 1.8$ & $349.3 \pm 1.1$ & $2.78 \pm 0.11$ \\
\hline \multirow{2}{*}{2008 Jan 15} & Keck/NIRC2 (masking) & $K_{S}$ & $144.6 \pm 2.3$ & $24.1 \pm 0.7$ & $2.97 \pm 0.11$ \\
\hline & Keck/NIRC $2^{\mathrm{a}}$ & $K_{S}$ & $146 \pm 4$ & $25.1 \pm 0.5$ & $2.70 \pm 0.12$ \\
\hline \multirow[t]{4}{*}{2009 Jan 23} & Keck/NIRC $2^{\mathrm{a}}$ & $K_{S}$ & $204.0 \pm 1.5$ & $53.8 \pm 0.3$ & $2.80 \pm 0.03$ \\
\hline & Keck/NIRC2 & $H$ & $202.0 \pm 2.4$ & $53.9 \pm 0.5$ & $2.96 \pm 0.05$ \\
\hline & Keck/NIRC2 & $J$ & $196 \pm 5$ & $53.9 \pm 0.8$ & $3.12 \pm 0.08$ \\
\hline & Keck/NIRC2 & $L^{\prime}$ & $200 \pm 3$ & $53.3 \pm 0.4$ & $1.92 \pm 0.06$ \\
\hline
\end{tabular}

Note. ${ }^{a}$ Used in the orbit fit.

that targeted brown dwarfs with HST/WFPC2-PC1 (GO-8563, PI Kirkpatrick; GO-8581, PI Reid; and GO-8146, PI Reid). We only used stars that had equivalent or higher signal-to-noise ratio $(\mathrm{S} / \mathrm{N})$ than the science data so that we could degrade the $\mathrm{S} / \mathrm{N}$ of the library images to match the science data. We also restricted ourselves to observations consisting of two or more images to allow robust rejection of cosmic rays. This library served to simulate the unsaturated images of LHS 2397aA and LHS $2397 \mathrm{aB}$. To simulate the saturated images of LHS 2397aA, we used other targets from the same program that had also been intentionally saturated to search for faint companions. We used the five targets closest in apparent flux to LHS 2397aA (LHS 2924, BRI 0021-0214, TVLM 513-46546, LP 412-31, and LHS 2243), which were $\lesssim 30 \%$ different in flux as measured by our PSF-fitting routine. We did not scale these images to match LHS 2397aA's flux since it is impossible to scale the saturated pixels. We added images of single stars from our library to these saturated images after scaling them down and adding Poisson noise to match the S/N of LHS $2397 \mathrm{aB}$. We shifted these images by an integer number of pixels that best reproduced the observed separation of $(+4.1,+4.4)$ pixels. By applying our PSF-fitting routine to these images, we were able to assess the position and flux measurements of a faint companion made in the presence of a saturated primary.

From these Monte Carlo simulations, we derived rms errors in measured $(x, y)$ positions of $(0.05,0.06)$ pixels and $(0.07$, 0.07 ) pixels for the $300 \mathrm{~s}$ and $1000 \mathrm{~s}$ images, respectively, with no significant systematic offsets. ${ }^{6}$ For the fluxes, the ratio of the input to best-fit value was $1.05 \pm 0.05$ for the $300 \mathrm{~s}$ image and $1.07 \pm 0.11$ for the $1000 \mathrm{~s}$ images. In addition, we were able to assess the intrinsic uncertainty in our TinyTim PSFfitting routine (i.e., the error when not in the presence of a saturated star) by comparing each undithered pair of PSF library images taken in the same orbit. From this ensemble of singlestar position and flux measurements, we found that the scatter in both $x$ and $y$ position measurements was 0.03 pixels, and the scatter in peak flux was a factor of 0.06 . We adopt these errors for the position and flux of LHS 2397aA measured in the $2 \mathrm{~s}$ image, and we adopt the errors from the Monte Carlo for the positions and fluxes of LHS $2397 \mathrm{aB}$ measured in the longer, saturated-

\footnotetext{
6 We found significant statistical correlation between the truth-minus-fitted offsets of the $x$ and $y$ positions $\left(\sigma_{x y}^{2} / \sigma_{x} \sigma_{y} \approx 0.6\right)$. We accounted for this covariance when propagating errors to compute the uncertainty in the separation and P.A.
}

primary exposures. This results in a separation uncertainty of 0.08 pixels (4 mas), a P.A. uncertainty of 0.8 , and a flux ratio uncertainty of $0.08 \mathrm{mag}$.

The drift correction we derived is thus quite significant compared to our derived positional uncertainties. However, if we do not apply this drift correction, the scatter in the measured position of LHS $2397 \mathrm{aB}$ is twice as large as predicted from our Monte Carlo simulations. As discussed in Section 3.1, the orbit determination results in an almost identical dynamical mass if this single $H S T$ epoch is excluded. Thus, our derived relative astrometry for the $H S T$ data is consistent with the orbit as determined from all other available astrometry.

\subsection{Keck/NIRC2 LGS}

We have monitored LHS 2397aAB using the LGS AO system at the Keck II Telescope on Mauna Kea, Hawaii (Wizinowich et al. 2006; van Dam et al. 2006). We used the facility nearinfrared camera NIRC2 in its narrow field-of-view mode because this provides the finest pixel scale. At each epoch, we obtained data in one or more filters covering the standard atmospheric windows from the Mauna Kea Observatories (MKO) filter consortium (Simons \& Tokunaga 2002; Tokunaga et al. 2002). We also obtained data with the $\mathrm{CH}_{4}$ s filter, positioned shortward of a methane absorption band $\left(\lambda_{c}=1.592 \mu \mathrm{m}\right.$, $\Delta \lambda=0.126 \mu \mathrm{m}$ ), to enable more robust photometric discrimination in the spectral type estimate of LHS $2397 \mathrm{aB}$.

The LGS provided the wavefront reference source for AO correction, with the exception of tip-tilt motion. The LGS brightness, as measured by the flux incident on the $\mathrm{AO}$ wavefront sensor, was equivalent to a $V \approx 10.4-10.7 \mathrm{mag}$ star. The tip-tilt correction and quasi-static changes in the image of the LGS as seen by the wavefront sensor were measured contemporaneously by a second, lower bandwidth wavefront sensor monitoring LHS 2397a, which saw the equivalent of a $R \approx 15.7-16.2$ mag star.

On each observing run, we obtained dithered images, offsetting the telescope by a few arcseconds between every 2 5 images. The sodium LGS was positioned at the center of the NIRC2 field of view for all observations. The images were reduced in a standard fashion. We constructed flat fields from

\footnotetext{
7 The Keck LGS AO tip-tilt sensor is very red sensitive, so a red source such as LHS 2397a appears much brighter than would otherwise be expected $(R=$ $17.9 \mathrm{mag}, I=14.6 \mathrm{mag}$; Monet et al. 2003). For late-M dwarfs, we have found that the Keck tip-tilt sensor generally sees the equivalent of an $(I+1.0)$ mag star.
} 

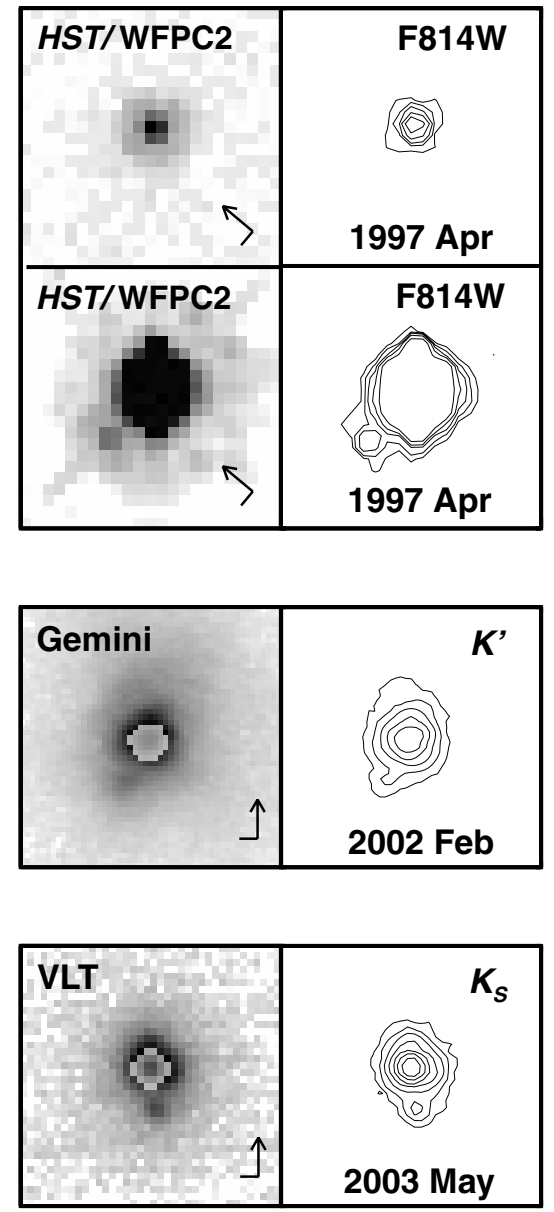

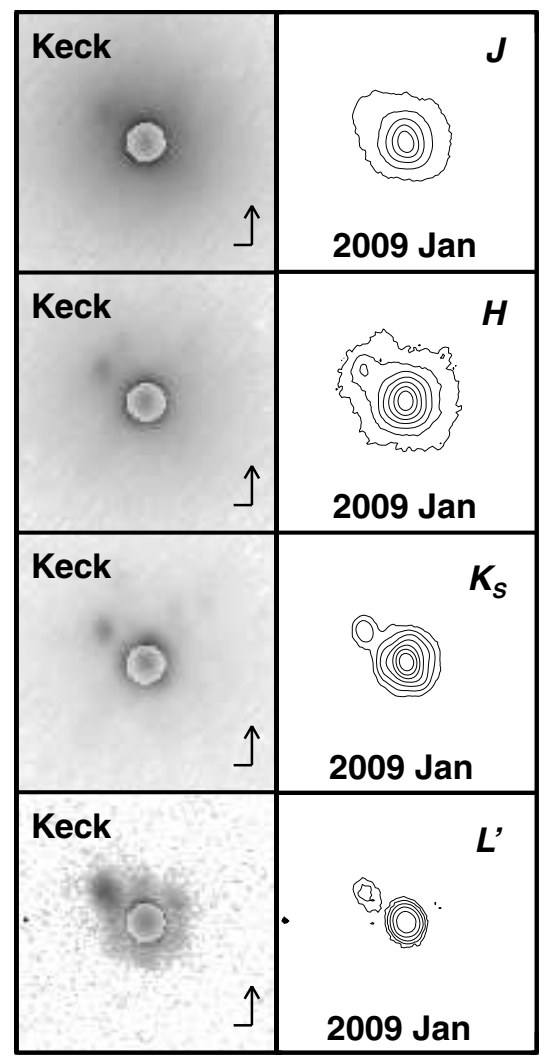

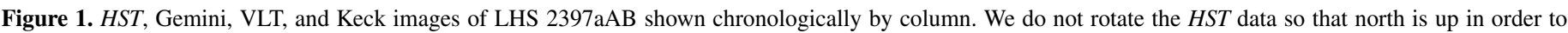

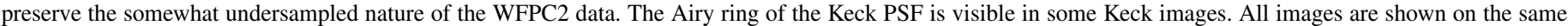

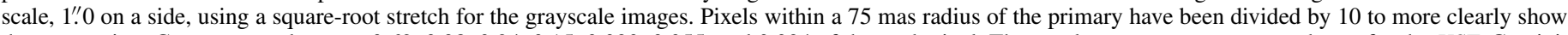

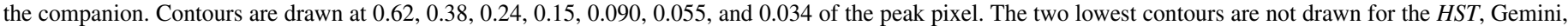
VLT (2006), and Keck $J$ - and $L^{\prime}$-band images.

the differences of images of the telescope dome interior with and without continuum lamp illumination. Then we created a master sky frame and subtracted it from the individual images. For $L^{\prime}$-band images, the sky subtraction was done by pairwise subtraction of consecutive images. Sky-subtracted images were registered and stacked to form a final mosaic, though all the results described here were based on analysis of the individual images. Typical images from each data set are shown in Figure 1. Outlier images with much poorer full width at half-maxima (FWHMs) and/or Strehl ratios were excluded from the analysis. We used the publicly available IDL routine NIRC2STREHL ${ }^{8}$ to estimate the Strehl and FWHM at each epoch, and in Table 1 the mean and standard deviation of these values over each image set are reported. The variation in the Strehl ratio and FWHM between epochs is due to different seeing conditions.

To determine the relative positions and fluxes of LHS 2397aA and LHS $2397 \mathrm{aB}$ in the imaging data, we used a simple analytic representation of the PSF to deblend the two components. The model was the sum of three elliptical Gaussians in which each Gaussian component was allowed to have a different FWHM and normalization, but all components had the same ellipticity and semimajor axis P.A. The best-fit parameters were found by a Levenberg-Marquardt least-squares minimization in which all

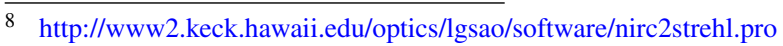

pixels were weighted equally. This fitting procedure yielded a set of measurements of the projected separation, P.A., and flux ratio for LHS $2397 \mathrm{aAB}$. We used the astrometric calibration from Ghez et al. (2008), with a pixel scale of $9.963 \pm 0.005$ mas pixel $^{-1}$ and an orientation for the detector's $+y$-axis of $+0.13 \pm$ 0.02 east of north. We applied the distortion correction developed by B. Cameron (2007, private communication) to the astrometry, which changed the results well below the $1 \sigma$ level. We also computed the change in the relative astrometry due to differential chromatic refraction (DCR) in the same manner as Dupuy et al. (2009) but did not apply this correction to our measurements because we found that it induces only a $<0.1 \sigma$ change.

To assess systematic errors in our PSF-fitting procedure, we also applied it to simulated Keck images of LHS 2397aAB. We scaled down each individual image until the primary flux matched the flux from the faint companion. In this scaled image, the companion flux is at the level of the noise. We then shifted and added this image to the original image, matching the binary separation of LHS $2397 \mathrm{aAB}$ but avoiding P.A.s within $\pm 60^{\circ}$ of LHS $2397 \mathrm{aB}$, which were masked out in the original image. After running our PSF-fitting routine on these images, the resulting scatter in the truth-minus-fitted parameters was comparable to or somewhat smaller than the rms scatter of the individual measurements $(\lesssim 0.5 \sigma$ different $)$, thus indicating that the rms scatter is a reasonable assessment of the errors. The Monte Carlo simulations also typically indicated significant systematic 
offsets $(\gtrsim 1 \sigma)$ in the astrometry and also the flux ratio (except for the 2009 data). However, we did not apply these offsets to any of the parameters derived from PSF fitting as these offsets have an insignificant impact on the resulting mass estimate (see Section 3.1). They also bring the $K_{S}$-band flux ratios derived from Keck imaging out of agreement with the ensemble of flux ratio measurements. This is not unexpected since the simulated images cannot exactly reproduce the science data, which have a somewhat asymmetric PSF and speckles that cause distinct systematic offsets at different separations and P.A.s.

In 2007 and 2008, data were also obtained using the ninehole non-redundant aperture mask installed in the filter wheel of NIRC2 (Tuthill et al. 2006). The data were taken in two dither positions separated by 3.5 , with four to six 50 s exposures taken at each dither position. With an integrated $K$-band brightness of 10.7 mag, LHS 2397a was fainter than typical targets previously observed with adaptive optics and the aperture mask, but it was still bright enough not to be limited by readout or background noise. Typical interferograms are shown in Figure 2. The pipeline used to reduce the aperture masking data was similar to that used in previous papers containing NIRC2 masking data (Ireland et al. 2008; Kraus et al. 2008; Ireland \& Kraus 2008), except that no comparable single star was observed for calibration of the closure phases or squared visibilities. For this reason, we chose to only fit to the closure phases, which from previous experience with NIRC2 never show an rms scatter of more than $3^{\circ}$ on bright calibrators in $H$ or $K$ band. Use of squared visibilities would have required a model of the fringe decorrelation due to an imperfect $\mathrm{AO}$ system, a complexity we chose not to tackle. The resulting closure phases from our observations are shown in Figure 2. The closure phases are intrinsically a four-dimensional function (the phase of the bispectrum, e.g., Lohmann et al. 1983), so they are difficult to present on a two-dimensional plot. Note that the closure phases are a linear function of contrast for contrast ratios larger than $\sim 5: 1$, so for example if the companion were twice as bright, then all the measured and model closure phases would be doubled.

The closure phase uncertainties were initially approximated by the standard error of the mean calculated from the scatter among individual exposures. The uncertainties were subsequently increased by adding a calibration error in quadrature so that the resulting reduced $\chi^{2}$ of the fit was 1.0. Although we fit all 84 closure phases from the nine-hole mask, only 28 of these are formally independent. To correctly account for this non-diagonal covariance matrix in our binary fitting, we scaled the errors in the least-squares fit to the data by $\sqrt{84 / 28}$. This process has been validated both by a comparison to fits using full covariance matrices (Kraus et al. 2008) and by orbit fits using mixed data that resulted in a reduced $\chi^{2}$ consistent with unity, where the orbit fit had many degrees of freedom (e.g., Martinache et al. 2007).

In Figure 2, it can be seen that binary is clearly detected at $>3 \sigma$ in many individual closure triangles for the two $K_{S^{-}}$ band detections. The $\mathrm{CH}_{4} \mathrm{~s}$-band detection has only one closure triangle above $3 \sigma$ but nonetheless has a very clear and unique fit to all triangles taken together that is consistent with the other data from the same epoch.

The astrometry and flux ratios derived from the Keck data are given in Table 2. The different data sets at each epoch, both aperture masking and direct imaging, give consistent measurements of the binary parameters. When fitting the orbit, we adopt the measurements from the single data set at each

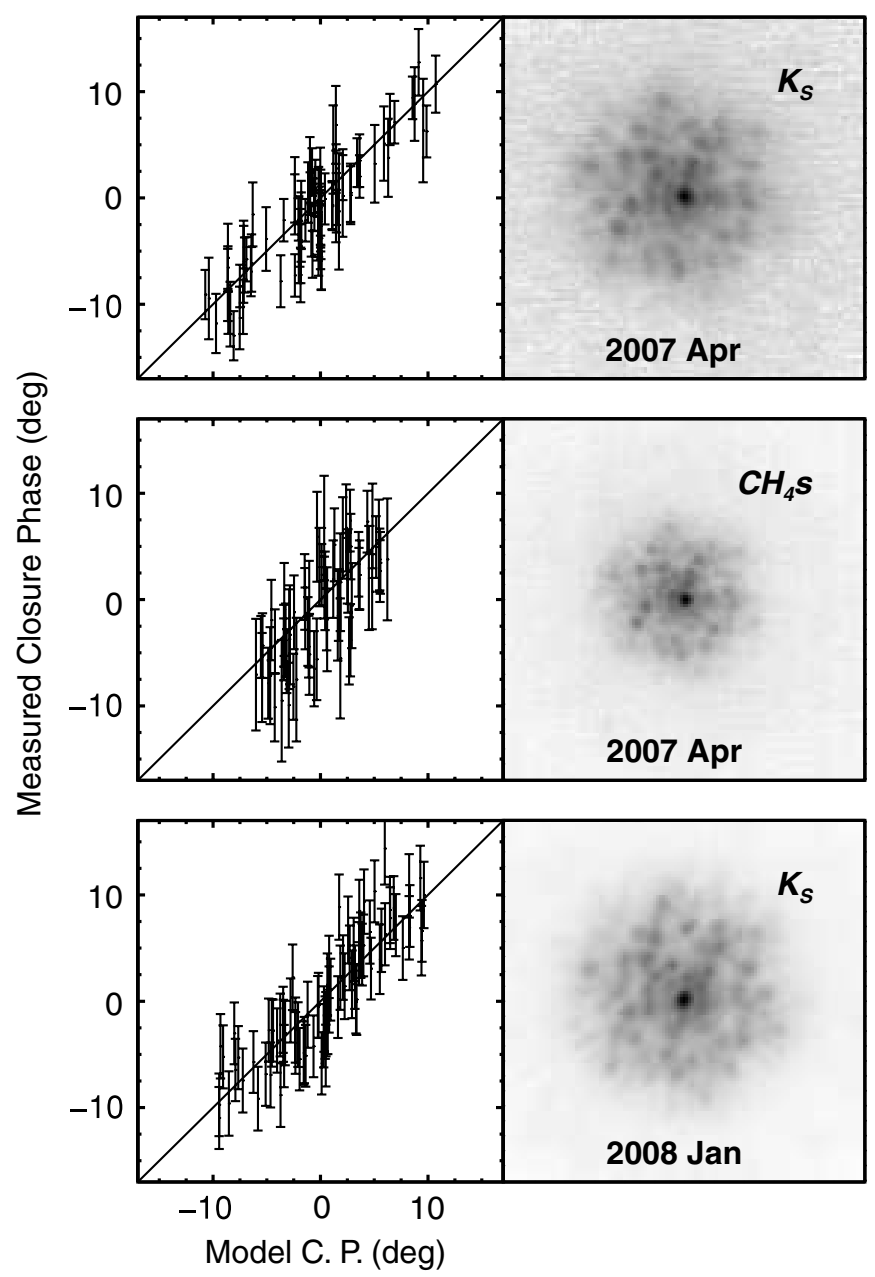

Figure 2. Keck interferograms of LHS 2397aAB obtained using NIRC2's ninehole aperture mask (square-root stretch). The left panels show the modeled vs. measured closure phases (C.P.) at each epoch. The binary parameters were derived from the modeled closure phases, and their errors were assessed in a Monte Carlo fashion that accounted for the measured closure phase errors.

epoch with the smallest astrometric errors. For the 2008 data, the error in separation is smaller for the masking data, while the P.A. error is smaller in the imaging data. Because the binary separation is very close to the outer limit at which masking observations are unambiguous in the 2008 data $(\approx 150$ mas; imposed by the smallest non-redundant baselines in the mask), we conservatively chose to adopt imaging astrometry for the 2008 epoch when fitting the orbit. Regardless of which $K_{S}$-band data set we use at each epoch, the resulting mass estimate is not significantly changed, as discussed in Section 3.1.

\subsection{Gemini/Hokupa'a}

LHS $2397 \mathrm{aAB}$ was imaged on UT 2002 February 7 by the Hokupa'a curvature AO system at the Gemini-North Telescope on Mauna Kea, Hawai'i. We retrieved these raw data from the Gemini science archive and registered, sky-subtracted, and performed cosmic-ray rejection on the images. Figure 1 shows a typical image from of one of the $15 K^{\prime}$-band $3 \mathrm{~s}$ exposures which were used to derive the astrometry for LHS 2397aAB. Analysis of these data has previously been presented by Freed et al. (2003); however, astrometric errors were not derived in that work, so we conducted our own analysis of these data.

We used the same analytic PSF-fitting routine as for the Keck data to fit the Gemini images of LHS 2397aAB. Adopting the 
nominal instrument pixel scale of $19.98 \pm 0.08$ mas pixel $^{-1}$, we found a separation of $205 \pm 8$ mas, where the uncertainty is the standard deviation of measurements from individual dithers. This is in good agreement with the 207 mas separation reported by Freed et al. (2003). However, the P.A. we find (206.8 \pm 2.4 ) is significantly different from the 152.0 P.A. reported by Freed et al. (2003). This must be due to an inaccurate reporting of the orientation of the camera in the header of the archival data, which indicates that the P.A. of the $+y$-axis is $0^{\circ}$. Such a discrepancy was previously seen in our work with archival Gemini/Hokupa'a data of HD 130948BC and Gl 569Bab (Dupuy et al. 2009). Thus, we adopt the published value of the P.A., but not its quoted error, for our analysis.

To assess systematic errors in the Gemini astrometry, we simulated many Gemini images of LHS 2397aAB in the same fashion as the Keck data and fit each one with our analytic PSF model. The resulting scatter in the truth-minus-fitted parameters was 14 mas in separation and $4^{\circ}$ in P.A., with no significant systematic offsets. These errors are much larger than the rms scatter of the individual measurements, which is likely because the PSF in the science data is somewhat asymmetric so that the offset between the best-fit and input parameters varies widely, depending on the binary P.A., and by averaging over many binary P.A.s we overestimate the scatter. However, as shown in Section 3.1, whether we adopt the rms scatter or the Monte Carlo errors only affects the $\chi^{2}$ of the orbit and not the resulting mass estimate. In fact, even if the Gemini epoch is excluded from the orbit fit the resulting mass estimate changes insignificantly.

\subsection{VLT/NACO}

We retrieved archival images of LHS 2397aAB obtained with the VLT at Paranal Observatory on UT 2003 May 31 and 2006 January 15. These data were taken with the NACO adaptive optics system (Lenzen et al. 2003; Rousset et al. 2003) using the N90C10 dichroic at both epochs and the S27 and S13 cameras in 2003 and 2006, respectively. The nominal pixel scales of these cameras are $27.053 \pm 0.019$ mas pixel $^{-1}$ and $13.221 \pm 0.017$ mas pixel $^{-1} .9$ We registered, sky-subtracted, and performed cosmic-ray rejection on the raw archival images. The 2003 data comprise five $K_{S}$-band $5 \mathrm{~s}$ exposures, and the 2006 data comprise $32 K_{S}$-band 10 s exposures. Typical images from each data set are shown in Figure 1.

We used the same analytic PSF-fitting routine as for the Keck data to fit the VLT images of LHS 2397aAB. From the 2003 VLT data we derived a separation of $168 \pm 8$ mas, a P.A. of $188.6 \pm 1.2$, and a $K_{S}$-band flux ratio of $2.75 \pm 0.16 \mathrm{mag}$. From the 2006 VLT data we derived a separation of $129 \pm 5$ mas, a P.A. of $276.2 \pm 1.4$, and a $K_{S}$-band flux ratio of $2.84 \pm 0.16 \mathrm{mag}$. The quoted uncertainty is the standard deviation of measurements from individual dithers. To assess any additional systematic errors in the VLT astrometry, we simulated many VLT images of LHS 2397aAB in the same fashion as the Gemini and Keck data. These simulations yielded equivalent errors to the rms scatter quoted above $(\lesssim 0.2 \sigma$ different $)$, and thus the quoted errors are a good representation of the uncertainties.

\section{RESULTS}

\subsection{Orbit Determination and Dynamical Mass}

The orbit of LHS $2397 \mathrm{aAB}$ is very well constrained as our observations cover most of the orbital period. In order to search

\footnotetext{
9 http://www.eso.org/sci/facilities/paranal/instruments/naco/ doc/VLT-MAN-ESO-14200-2761_v83.3.pdf
}

for the influence of parameter degeneracies in our orbit fit and determine robust confidence limits on the orbital parameters, we used a Markov Chain Monte Carlo (MCMC) technique (e.g., Bremaud 1999) for orbit fitting, in addition to a gradient descent technique. In short, the MCMC method constructs a series of steps through the model parameter space such that the resulting set of values (the "chain") is asymptotically equivalent to the posterior probability distribution of the parameters being sought. The code that performed the MCMC fit is described in detail in the study of 2MASS J1534-2952AB by Liu et al. (2008). Chains all had lengths of $2 \times 10^{8}$ steps, and the correlation length of our most correlated chain, as defined by Tegmark et al. (2004), was 230 for the argument of periastron. This gives an effective length of the chain of $8.7 \times 10^{5}$, which in turn gives statistical uncertainties in the parameter errors of about $1 / \sqrt{8.7 \times 10^{5}}=0.11 \%$, i.e., negligible.

We used uniform priors in period $(P)$, semimajor axis $(a)$, P.A. of the ascending node $(\Omega)$, argument of periastron $(\omega)$, and time of periastron passage $\left(T_{0}\right)$. We used a prior in inclination proportional to $\sin (i)$ (i.e., random orbital orientation) and an eccentricity prior of $f(e)=2 e$ (e.g., see Duquennoy \& Mayor 1991). Figure 3 shows the resulting MCMC probability distributions for the seven orbital parameters of LHS 2397aAB. The best-fit parameters and their confidence limits are given in Table 3, and the best-fit orbit is shown in Figures 4 and 5. The reduced $\chi^{2}$ of the orbital solution is 1.04 , with 7 degrees of freedom.

Applying Kepler's Third Law to the period and semimajor axis distributions gives the posterior probability distribution for the total mass of LHS $2397 \mathrm{aAB}$, with a median of $0.146 M_{\odot}$, a standard deviation of $0.004 M_{\odot}$, and 68.3(95.4)\% confidence limits of ${ }_{-0.004}^{+0.004}\left({ }_{-0.008}^{+0.008}\right) M_{\odot}$ (Figure 6). The MCMC probability distribution of the total mass does not include the uncertainty in the parallax $(3.0 \%)$, which by simple propagation of errors would contribute an additional $9.0 \%$ uncertainty in mass. In fact, since the MCMC-derived mass distribution is slightly asymmetric, we account for this additional error by randomly drawing a normally distributed parallax value for each step in the chain, which we then used to compute the total mass. The resulting mass distribution is nearly indistinguishable from Gaussian (Figure 6). Our final determination of the total mass is $0.146_{-0.013}^{+0.015}\left({ }_{-0.024}^{+0.031}\right) M_{\odot}$ at $68.3(95.4) \%$ confidence.

As an independent verification of our MCMC results, we also fit the orbit of LHS 2397aAB using the linearized least-squares routine ORBIT (described in Forveille et al. 1999). All of the orbital parameters and their errors are consistent between the ORBIT and MCMC results, as expected for well-constrained parameters with nearly Gaussian probability distributions. The $\chi^{2}$ and total mass of the ORBIT solution and the MCMC solution were identical.

From the visual orbit alone there is a $180^{\circ}$ ambiguity in the P.A. of the ascending node $(\Omega)$, and correspondingly $\omega$, that can only be resolved by radial velocity measurements. Basri \& Reiners (2006) measured the radial velocity of LHS 2397a at two epochs (UT 1995 Mar 12 and 2002 May 19) in the optical where LHS $2397 \mathrm{aB}$ is essentially invisible. For a mass ratio of 0.7 (Section 4.2), the expected difference in the radial velocity of the primary between these two epochs is $\pm 1.8 \mathrm{~km} \mathrm{~s}^{-1}$, where the unknown sign reflects the $180^{\circ}$ ambiguity in $\Omega$. Basri \& Reiners (2006) measured a velocity difference of $+1.1 \pm 1.6 \mathrm{~km}$ $\mathrm{s}^{-1}$, which is consistent with $+1.8 \mathrm{~km} \mathrm{~s}^{-1}$ but discrepant with $-1.8 \mathrm{~km} \mathrm{~s}^{-1}$ at $1.8 \sigma$. 

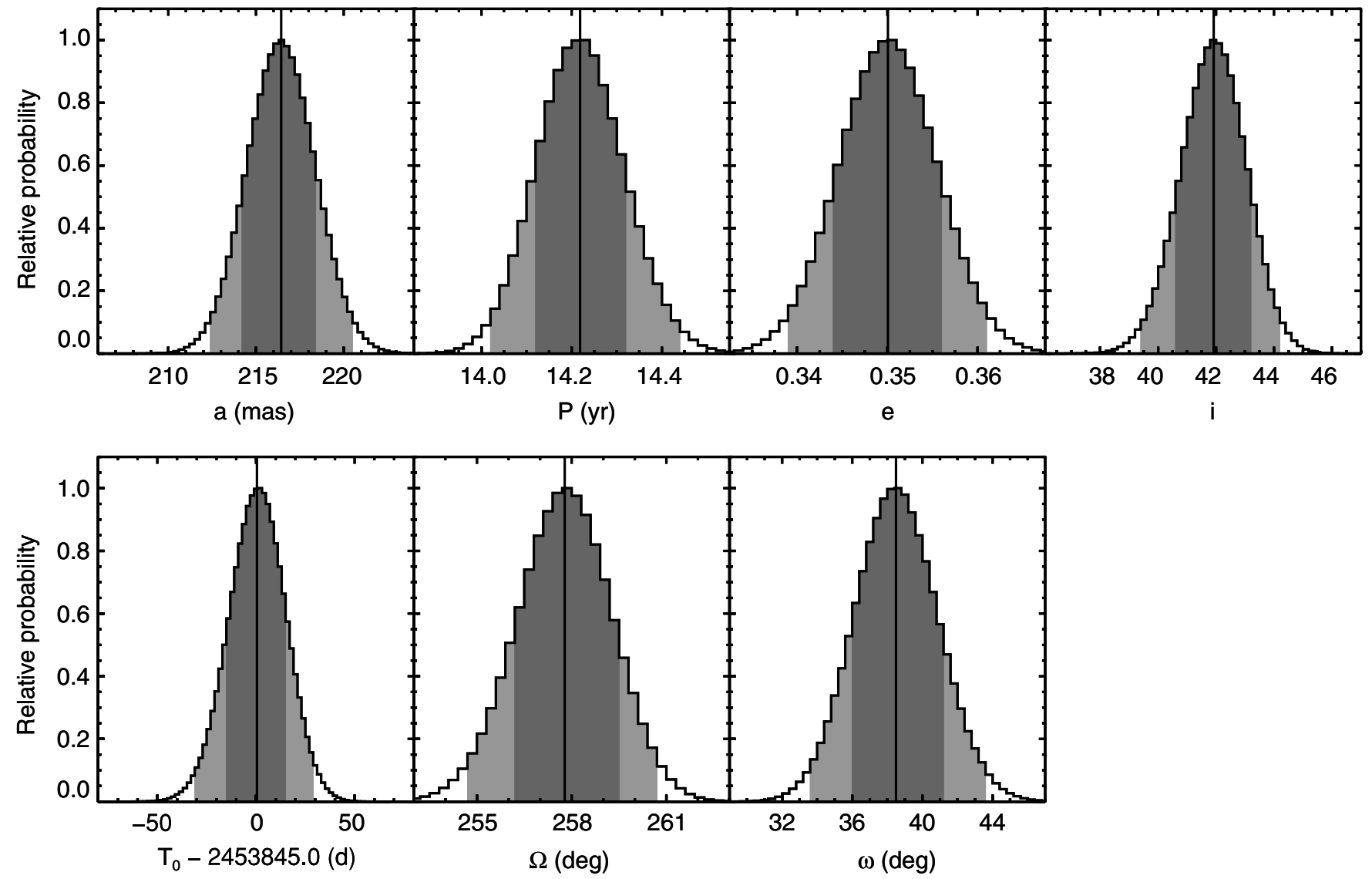

Figure 3. Probability distributions of all orbital parameters derived from the MCMC analysis: semimajor axis $(a)$, orbital period $(P)$, eccentricity $(e)$, inclination $(i)$, epoch of periastron $\left(T_{0}\right)$, P.A. of the ascending node $(\Omega)$, and argument of periastron $(\omega)$. Each histogram is shaded to indicate the $68.3 \%$ and $95.4 \%$ confidence regions, which correspond to $1 \sigma$ and $2 \sigma$ for a normal distribution, and the solid vertical lines represent the median values. Note that $T_{0}$ is shown in days since UT 2006 Apr 19 12:00 for clarity.

Table 3

Derived Orbital Parameters for LHS 2397aAB

\begin{tabular}{|c|c|c|c|c|}
\hline \multirow[t]{2}{*}{ Parameter } & \multicolumn{3}{|c|}{ MCMC } & \multirow[t]{2}{*}{ ORBIT $^{\mathrm{a}}$} \\
\hline & Median & $68.3 \%$ c.l. & $95.4 \%$ c.l. & \\
\hline Semimajor axis $a$ (mas) & 216.4 & $-1.9,2.0$ & $-3.8,3.9$ & $216.5 \pm 2.0$ \\
\hline Orbital period $P(\mathrm{yr})$ & 14.22 & $-0.10,0.10$ & $-0.19,0.20$ & $14.22 \pm 0.10$ \\
\hline Eccentricity $e$ & 0.350 & $-0.005,0.005$ & $-0.011,0.011$ & $0.345 \pm 0.005$ \\
\hline Inclination $i\left(^{\circ}\right)$ & 41.9 & $-1.1,1.1$ & $-2.3,2.2$ & $42.0 \pm 1.1$ \\
\hline Time of periastron passage $T_{0}-2453845.0^{\mathrm{b}}(\mathrm{JD})$ & 0 & $-15,14$ & $-30,28$ & $0 \pm 15$ \\
\hline P.A. of the ascending node $\Omega\left({ }^{\circ}\right)$ & 257.8 & $-1.5,1.5$ & $-3.1,2.9$ & $257.8 \pm 1.5$ \\
\hline Argument of periastron $\omega\left(^{\circ}\right)$ & 38.5 & $-2.3,2.4$ & $-4.6,4.8$ & $38.5 \pm 2.4$ \\
\hline Total mass $\left(M_{\odot}\right):$ fitted $^{\mathrm{c}}$ & 0.146 & $-0.004,0.004$ & $-0.008,0.008$ & $0.146 \pm 0.004$ \\
\hline Total mass $\left(M_{\odot}\right)$ : final $^{\mathrm{d}}$ & 0.146 & $-0.013,0.015$ & $-0.024,0.031$ & $0.146 \pm 0.14$ \\
\hline Reduced $\chi^{2}$ ( 7 degrees of freedom $)$ & 1.04 & $\cdots$ & $\cdots$ & 1.04 \\
\hline
\end{tabular}

Notes.

${ }^{\text {a }}$ The orbital parameters determined by ORBIT (Forveille et al. 1999), with their linearized $1 \sigma$ errors.

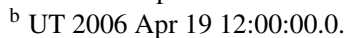

c The "fitted" total mass represents the direct results from fitting the observed orbital motion of the two components without accounting for the parallax error. For the linearized ORBIT error, the covariance between $P$ and $a$ is taken into account.

d The "final" total mass includes the additional 9.0\% error in the mass due to the error in the parallax. This final mass distribution is essentially Gaussian.

We have tested whether varying the input astrometry and corresponding uncertainties affects the orbit-fitting results using ORBIT. Regardless of what astrometry was used, the mass estimate and its uncertainty did not change significantly. We tested many permutations using ORBIT, such as using Keck imaging instead of masking astrometry or Monte Carlo instead of rms errors, and we found that the resulting mass estimates ranged from $0.142-0.150 M_{\odot}$ and uncertainties ranged from $0.003-0.006 M_{\odot}$. The $\chi^{2}$ of these different permutations ranged from 5-17 (7 degrees of freedom), so many of these scenarios are clearly not as favorable as our default solution that has a $\chi^{2}$ of 7.27. The permutations with lower $\chi^{2}$ simply correspond to the 


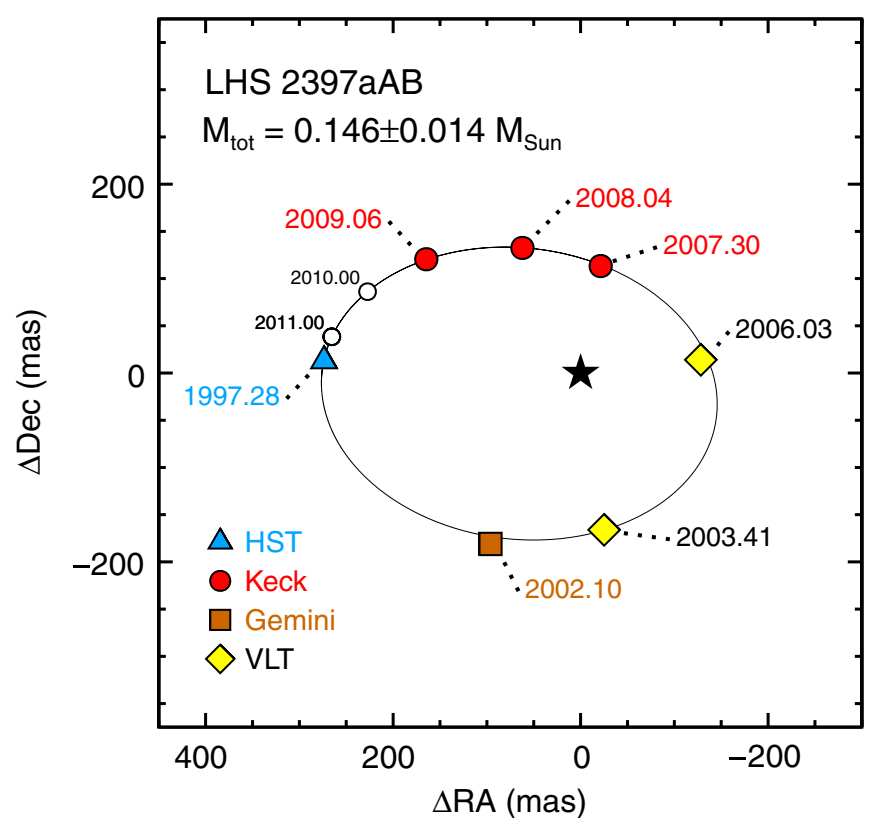

Figure 4. Relative astrometry for LHS $2397 \mathrm{aAB}$ along with the best-fitting orbit (reduced $\chi^{2}$ of 1.04 for 7 degrees of freedom). The empty circles show the predicted location of LHS $2397 \mathrm{aB}$ in the future. Error bars are comparable to or smaller than the plotting symbols. The orbit is very well constrained, so the uncertainty in the total mass is dominated by the $3.0 \%$ parallax error.

(A color version of this figure is available in the online journal.)

cases with somewhat larger assumed astrometric uncertainties, and these had essentially identical masses and mass errors as our default solution.

In fact, any single epoch can be excluded and the resulting dynamical mass changes by $<0.006 M_{\odot}$, with the uncertainty from the orbit alone increasing to only $\pm 0.006 M_{\odot}$, which is well below the error due to the parallax uncertainty. Furthermore, pairs of epochs can even be excluded without significantly changing the dynamical mass. For example, both of the HST and Gemini

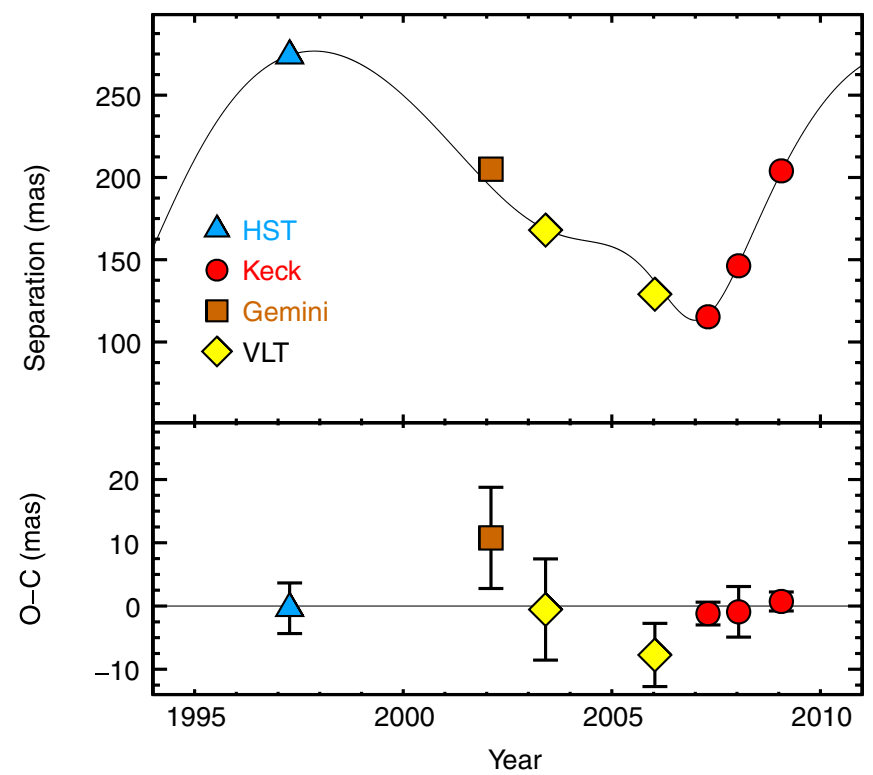

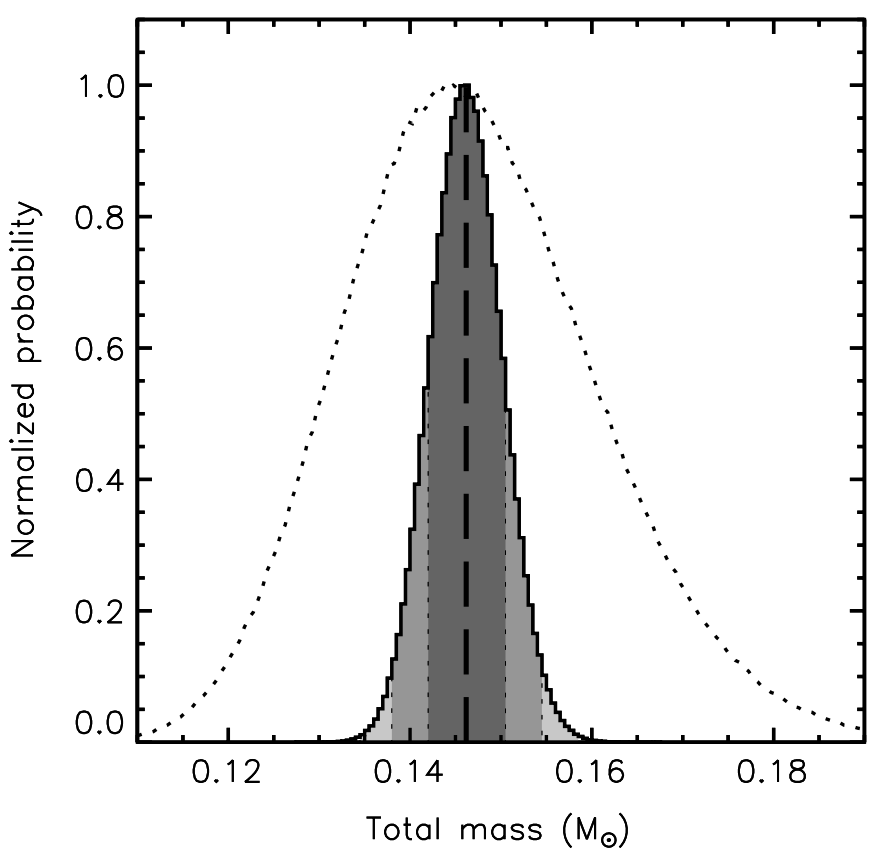

Figure 6. Probability distribution of the total mass of LHS 2397aAB resulting from our MCMC analysis. The histogram is shaded to indicate the $68.3 \%$, $95.4 \%$, and $99.7 \%$ confidence regions, which correspond to $1 \sigma, 2 \sigma$, and $3 \sigma$ for a normal distribution. The dashed line represents the median value of $0.146 M_{\odot}$. The standard deviation of the distribution is $0.004 M_{\odot}$. The dotted unshaded curve shows the final mass distribution after accounting for the additional $9.0 \%$ error due to the uncertainty in the parallax; the result is essentially Gaussian. The confidence limits for both distributions are given in Table 3 .

epochs or both of the VLT epochs can be excluded and the resulting dynamical mass changes by $<0.008 M_{\odot}$ and the error from the orbit alone increases to $0.013 M_{\odot}$ (i.e., comparable to the error due to the parallax uncertainty). Thus, our mass estimate for LHS 2397aAB and its uncertainty is insensitive to the input astrometry and the method of determining the measurement errors.

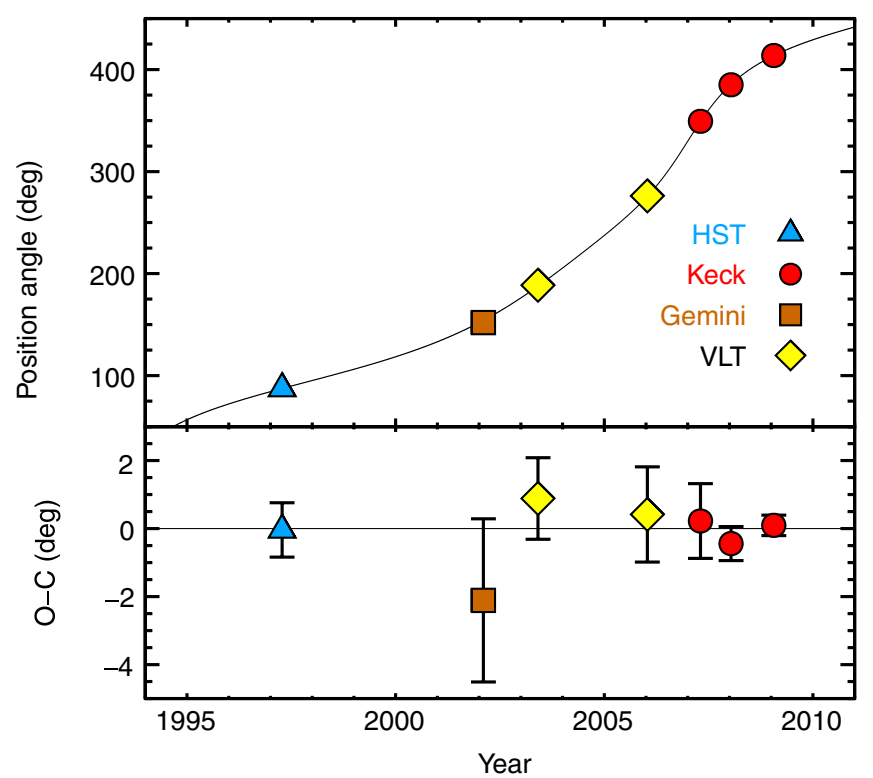

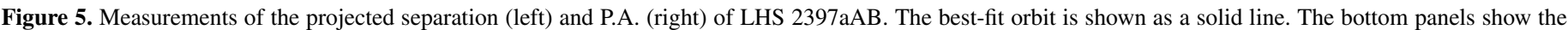

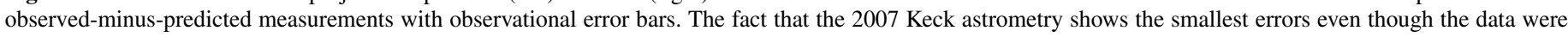

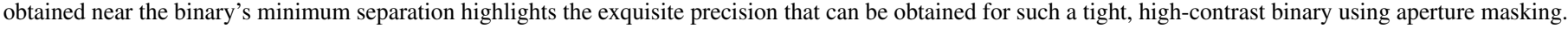

(A color version of this figure is available in the online journal.) 


\subsection{Spectral Types}

Spectral type determination for the components of LHS 2397aAB is impeded by the lack of resolved spectroscopy. However, the optical classification of M8 (Kirkpatrick et al. 1995 ) can be readily applied to the primary since the $I$-band flux ratio of $\approx 4$ mag indicates that LHS $2397 \mathrm{aB}$ contributes negligibly to the flux in the unresolved optical spectrum. The spectral type of LHS $2397 \mathrm{aB}$ must be estimated indirectly, and we have done so in a variety of ways.

The available IJHKL' colors of LHS $2397 \mathrm{aB}$ do not provide a strong constraint on the spectral type; however, they are consistent with late-L dwarfs (Dahn et al. 2002; Golimowski et al. 2004; Liebert \& Gizis 2006). Unfortunately, even if the colors of LHS $2397 \mathrm{aB}$ were measured perfectly, the dispersion among known L dwarfs limits a spectral type estimate from colors alone to at least \pm 2 subclasses.

We also used the empirical absolute magnitude relations of Liu et al. (2006) ${ }^{10}$ with known and suspected binaries removed to estimate the spectral type of LHS $2397 \mathrm{aB}$. We converted these relations from the MKO to the Two Micron All Sky Survey (2MASS) photometric system using the relations of Stephens \& Leggett (2004). In addition, we derived the $L^{\prime}$ band absolute magnitude relation for the same sample, though 7 of the 29 objects from Liu et al. (2006) could not be used as they did not have published $L^{\prime}$-band photometry (Leggett et al. 2002; Golimowski et al. 2004). The resulting polynomial fit was

$$
\begin{aligned}
M_{L^{\prime}}= & 9.759+\left(1.507 \times 10^{-1}\right) \times \mathrm{SpT}+\left(2.868 \times 10^{-2}\right) \times \mathrm{SpT}^{2} \\
& +\left(-3.674 \times 10^{-3}\right) \times \mathrm{SpT}^{3}+\left(1.338 \times 10^{-4}\right) \times \mathrm{SpT}^{4}
\end{aligned}
$$

with an rms scatter about this relation of 0.18 mag. By simply interpolating these absolute magnitude relations, the $K$-band photometry of LHS $2397 \mathrm{aB}$ gave a spectral type of L7.5 \pm 0.3 , the $H$-band photometry gave L6.6 \pm 0.3 , and the $L^{\prime}$-band photometry gave L7.4 \pm 0.5 . These quoted uncertainties only account for the photometric errors (i.e., the $1 \sigma$ spectral type range corresponds to the $1 \sigma$ range in photometry) and not scatter in the empirical relations. Because of the flattening of the absolute magnitude relation in $J$ band, we were unable to estimate a robust spectral type from the $J$-band photometry, although it is consistent with the spectral type ranges listed above.

We also employed a more sophisticated method for spectral type estimation that accounts for both the uncertainty in the absolute magnitude of LHS $2397 \mathrm{aB}$ and the scatter in the empirical relations. At each half-integer spectral type, we considered two Gaussian probability distributions: (1) the distribution of the observed absolute magnitude of LHS 2397aB and (2) the distribution of absolute magnitudes predicted by the empirical relation from the overall scatter in the relation as given by Liu et al. (2006). We multiplied these two distributions and integrated the result in order to obtain a measure of the relative probability of LHS $2397 \mathrm{aB}$ having that spectral type. If the two distributions overlapped substantially, then the integral was large, but if the two distributions were very discrepant the integral went to zero. Thus, we built an ensemble of relative probabilities which we adopted as the probability distribution of the spectral type of LHS $2397 \mathrm{aB}$ (Figure 7). We did this separately for each bandpass, and the derived spectral type and uncertainty was T0.9 $9_{-4.0}^{+3.5}$ for $J$ band, L7.2 $2_{-1.3}^{+2.2}$ for $H$ band, L7.6 $6_{-1.3}^{+1.4}$

\footnotetext{
10 These empirical relations are based on near-infrared spectral types.
}

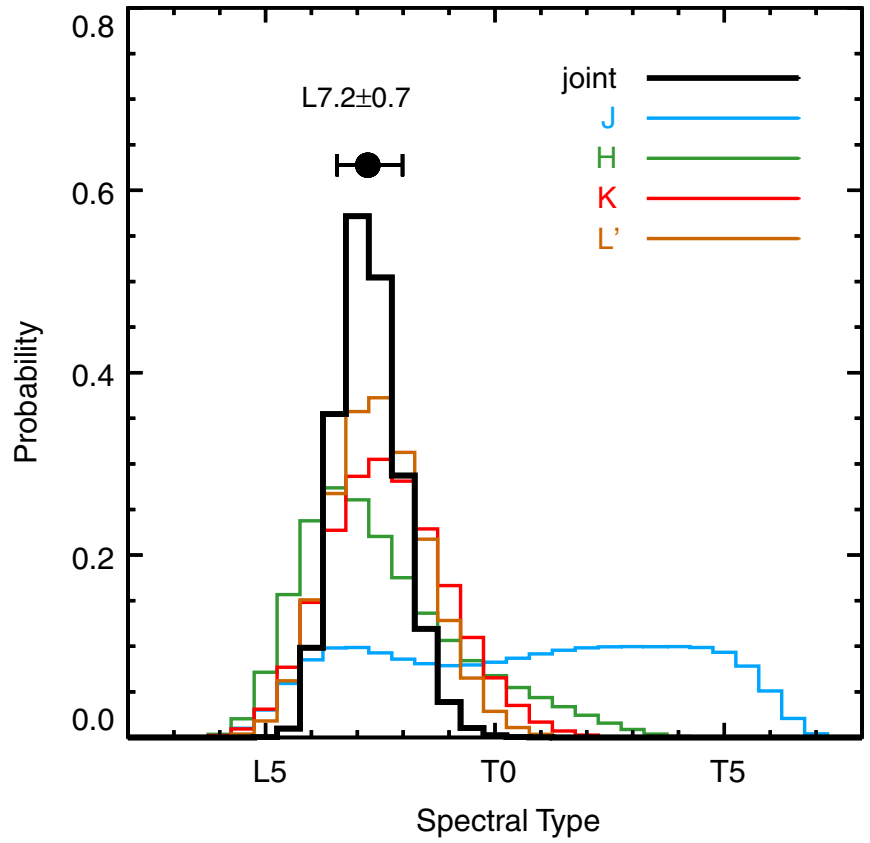

Figure 7. Final probability distribution of the spectral type of LHS 2397aB (solid black line), derived using the absolute magnitude relations of Liu et al. (2006), with suspected binaries removed, and our own $L^{\prime}$-band relation as described in the text. The joint probability distribution of the spectral type was derived from $J$-band, $H$-band, $K$-band, and $L^{\prime}$-band photometry (thin colored lines). The filled circle with error bars denotes the median and $1 \sigma$ confidence limits of this final spectral type distribution.

(A color version of this figure is available in the online journal.)

for $K$ band, and L7. $4_{-1.0}^{+1.1}$ for $L^{\prime}$ band (68.3\% confidence limits). Since each bandpass represents an independent constraint on the spectral type, we took the product of these different spectral type distributions to determine the final spectral type probability distribution (Figure 7). This is conceptually equivalent to taking the weighted mean of the spectral types listed above, and the result is L7.2 $\pm 0.7 .{ }^{11}$ Because of the good agreement between the spectral types inferred from different bandpasses, the final derived spectral type of LHS $2397 \mathrm{aB}$ is essentially the same with smaller errors.

We adopt the best spectral type estimates available: for LHS 2397aA this is M8 \pm 0.5 from the optical spectrum, and for LHS $2397 \mathrm{aB}$ we assign a spectral type of $\mathrm{L} 7 \pm 1$ from the absolute magnitude relations, where the uncertainty accounts for both the observational errors in the photometry and the scatter in the empirical relations.

\subsection{Bolometric Luminosities}

We computed bolometric luminosities for LHS 2397aAB in two different ways: (1) directly using integrated-light measurements of LHS 2397a and (2) using our resolved photometry for the individual binary components along with spectral templates. Kirkpatrick et al. (1995) measured the optical integrated-light spectrum of LHS $2397 \mathrm{a}(\approx 0.64-0.92 \mu \mathrm{m}) .{ }^{12}$ On 2008 June 27 UT we obtained a near-infrared spectrum of LHS 2397 a $(\approx 0.82-2.4 \mu \mathrm{m})$ using IRTF/SpeX (Rayner et al. 1998) in SXD mode $(R=1200)$, which we reduced using the SpeXtool software package (Vacca et al. 2003; Cushing et al. 2004).

\footnotetext{
11 If we use the Liu et al. (2006) empirical relations which do not exclude suspected binaries, the resulting spectral type of LHS $2397 \mathrm{aB}$ is L7.6 $6_{-0.9}^{+1.1}$.

12 Publicly available at http://www.dwarfarchives.org.
} 


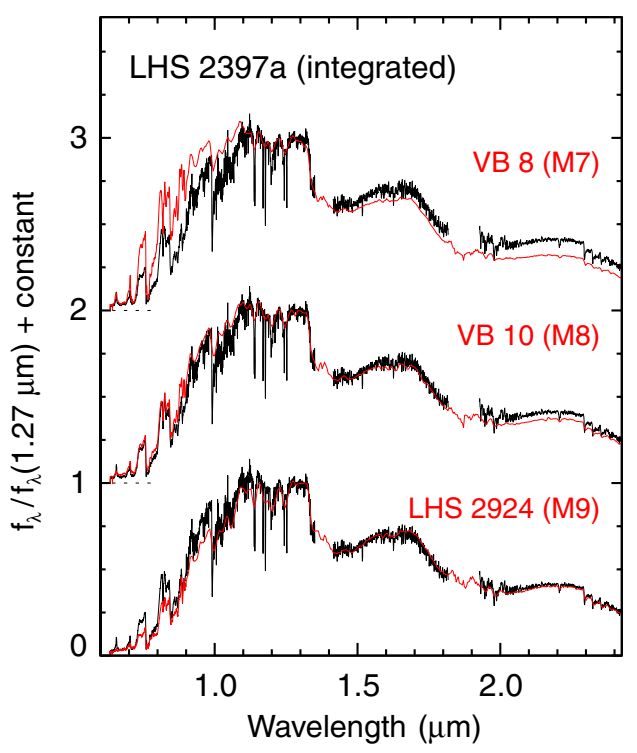

Figure 8. Integrated-light spectrum of LHS 2397a, with optical data $(<0.9 \mu \mathrm{m})$ taken from Kirkpatrick et al. (1995) and our near-infrared data obtained with IRTF/SpeX. Data for M dwarf spectral standards are shown for comparison (Henry \& Kirkpatrick 1990; Burgasser et al. 2004, 2008; Burgasser \& McElwain 2006). LHS 2397a is optically typed as M8, and its near-infrared ( $H$ and $K$ bands) spectrum is discrepant with the standard VB 10 due to additional flux from the very red L7 dwarf companion LHS $2397 \mathrm{aB}$.

(A color version of this figure is available in the online journal.)

We combined these spectra, using optical data below $0.9 \mu \mathrm{m}$ and our SpeX data above $0.9 \mu \mathrm{m}$. Figure 8 shows the resulting integrated-light spectrum of LHS 2397a, along with M dwarf spectral standards from Kirkpatrick et al. (1991). ${ }^{13}$

We derived a total bolometric luminosity of $\log \left(L_{\mathrm{bol}} / L_{\odot}\right)=$ $-3.33 \pm 0.03$ for LHS 2397a using the integrated-light spectrum $(0.6-2.4 \mu \mathrm{m}), L^{\prime}$-band $(3.8 \mu \mathrm{m})$ photometry from Leggett et al. (2002), and $24 \mu \mathrm{m}$ Spitzer/MIPS photometry from Gautier et al. (2007). We neglected any flux at shorter wavelengths, interpolated between gaps in the data, and extrapolated the flux beyond $24 \mu \mathrm{m}$ assuming a blackbody. We determined the luminosity error in a Monte Carlo fashion by adding randomly drawn noise to our data over many trials and computing the rms of the resulting luminosities.

For LHS 2397a, the SpeX spectrum and $L^{\prime}$-band photometry alone account for $98 \%$ of its luminosity. Thus, it is possible to accurately estimate the luminosities of the individual components using only our resolved $J H K L^{\prime}$ photometry and spectral templates to interpolate properly between bandpasses. We selected objects from the SpeX Prism Libraries ${ }^{14}$ that had similar colors to LHS 2397aA and LHS 2397aB and calibrated them to match our resolved $J H K$ photometry. In fact, we found that the exact choice of the templates had an insignificant impact on the resulting luminosity measurements. We integrated over the template spectra, neglecting flux at shorter wavelengths, interpolating the flux between the spectra and $L^{\prime}$ band, and extrapolating to longer wavelengths assuming a blackbody. Thus, we derived luminosities of $\log \left(L_{\text {bol }} / L_{\odot}\right)=-3.42 \pm 0.03$ for LHS $2397 \mathrm{aA}$ and $-4.52 \pm 0.03$ for LHS $2397 \mathrm{aB}$. The sum of these luminosities is $-3.39 \pm 0.03$, which is consistent with the

\footnotetext{
13 We combined optical and near-infrared data for the M dwarf spectral standards in the same way as LHS 2397a. The optical data are from Henry \& Kirkpatrick (1990), and the near-infrared data are SpeX prism spectra from Burgasser et al. $(2004,2008)$ and Burgasser \& McElwain (2006).

14 http://www.browndwarfs.org/spexprism/
}

$L_{\mathrm{bol}}$ derived from integrated-light data. The dominant source of error in these luminosities is the parallax, which is common to both components. Thus, the luminosities are correlated through the flux ratio, and in the following analysis we correctly account for this covariance.

\subsection{Age Constraints from Kinematics and Activity}

The space motion of LHS 2397a could provide a constraint on its age, especially if it were found to be kinematically associated with a population of known age (e.g., the thick disk). Using the proper motion and parallax from Monet et al. (1992) and a radial velocity of $34 \pm 2 \mathrm{~km} \mathrm{~s}^{-1}$ (Mohanty \& Basri 2003; Tinney \& Reid 1998), we computed the heliocentric velocity of LHS 2397a to be $(U, V, W)=(-26.5 \pm 0.8,-36.1 \pm 1.5$, $+12.5 \pm 1.4) \mathrm{km} \mathrm{s}^{-1} \cdot{ }^{15}$ We adopt the sign convention for $U$ that is positive toward the Galactic center and account for the errors in the parallax, proper motion, and radial velocity in a Monte Carlo fashion.

For comparison, we compiled all the radial velocities for objects with spectral types of M7 or later from Mohanty \& Basri (2003), Bailer-Jones (2004), and Zapatero Osorio et al. (2007) and computed space velocities for those objects with parallaxes and proper motions from Dahn et al. (2002), Monet et al. (1992), Tinney et al. (1995, 2003), Vrba et al. (2004), Tinney (1996), and Henry et al. (2006). The resulting heliocentric velocities are shown in Figure 9. The mean and rms scatter of the space velocity of this population is $(U, V, W)=(-18 \pm 37,-24 \pm$ $26,-5 \pm 15) \mathrm{km} \mathrm{s}^{-1}$, and LHS $2397 \mathrm{a}$ is only $1.3 \sigma$ away from the mean of this ellipsoid. Thus, the space motion of LHS 2397a is not significantly different from other ultracool dwarfs, implying an age consistent with the population of ultracool dwarfs as a whole. Several authors have attempted to estimate the age of this population, typically comparing the distribution of tangential velocities $\left(V_{\tan }\right.$, which requires only a proper motion and distance determination) to the well-studied nearby populations of FGKM stars. The resulting age for the population of ultracool dwarfs estimated in this way has been found to be 2-4 Gyr (Dahn et al. 2002; Faherty et al. 2009). ${ }^{16}$

We have also assessed LHS 2397a's membership in the Galactic populations of the thin disk (1-10 Gyr; e.g., Wood \& Oswalt 1998) and thick disk ( 10 Gyr; e.g., Ibukiyama \& Arimoto 2002) using the Besançon model of the Galaxy (Robin et al. 2003). Because the scale height of these structures are much larger than the measured distances to ultracool dwarfs, we found that a specialized model population for the volume within 1 square-degree on the sky and $5 \mathrm{pc}$ in the radial direction of LHS 2397a had essentially the same space motions as the model population of all objects within $50 \mathrm{pc}$ over the whole sky. Robin et al. (2003) describe the velocity ellipsoids of the Besançon model thin and thick disks (shown in Figure 9) and how these space motions are allowed to evolve over time due to dynamical heating. To determine membership probabilities of a

\footnotetext{
15 This is consistent with the heliocentric velocity derived by Freed et al. (2003), but inconsistent with the velocity computed by Tinney \& Reid (1998). It appears that their space velocity for LHS 2397a is discrepant by $\approx 2 \times(U, V, W)_{\odot}$ as a result of subtracting rather than adding the solar motion to the heliocentric velocities.

16 Zapatero Osorio et al. (2007) determined a somewhat younger age ( $\sim 1$ Gyr) for the population of ultracool dwarfs, based on the small sample of $\mathrm{L}$ and $\mathrm{T}$ dwarfs will full space velocities (21 objects). However, since $\mathrm{L}$ and $\mathrm{T}$ dwarfs span a wider range of masses than earlier type objects, a typical IMF that rises at lower masses will naturally increase the number of young objects in this sample, biasing a kinematically derived age (e.g., see Section 4.5 of Dahn et al. 2002).
} 

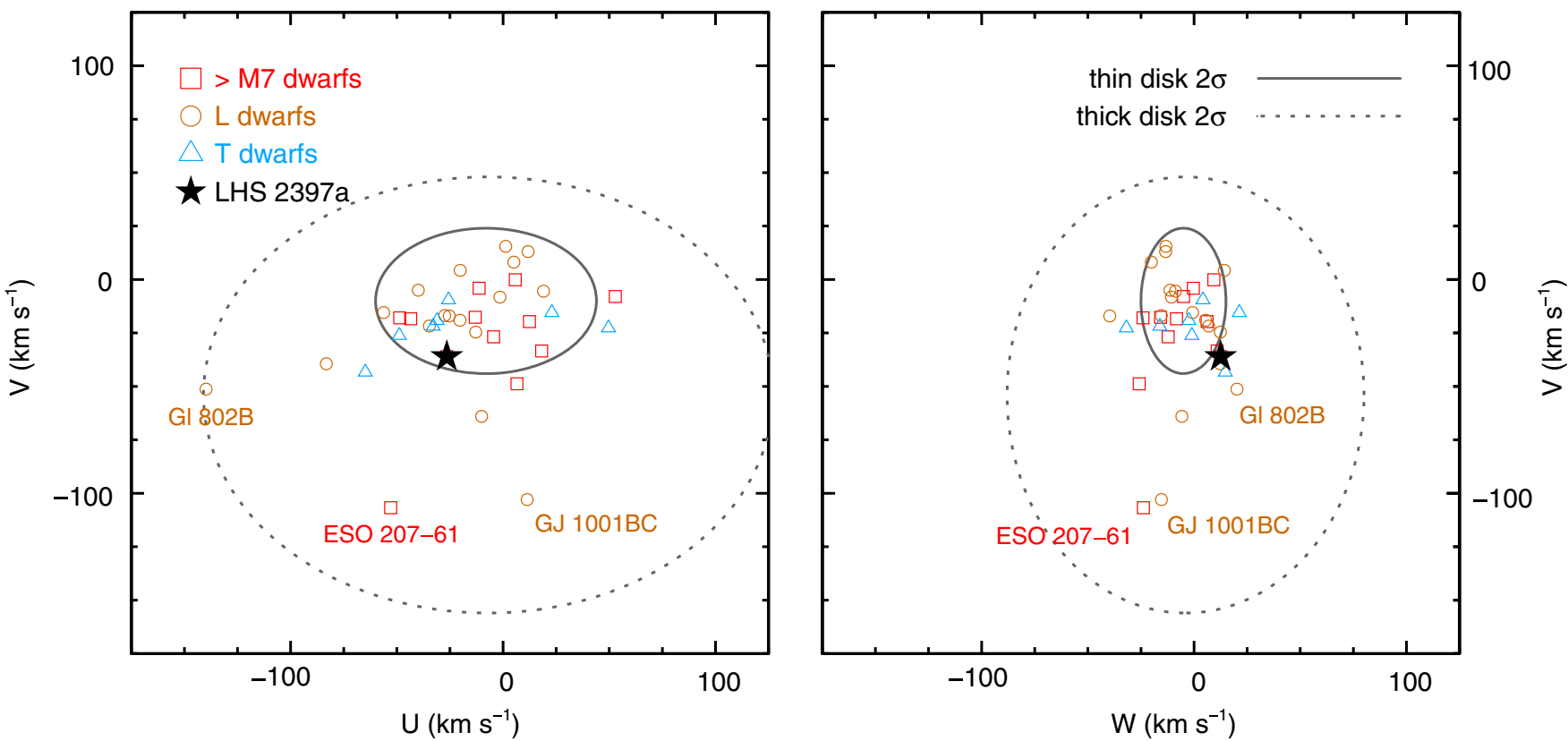

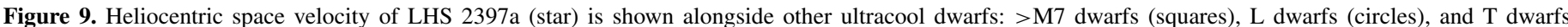

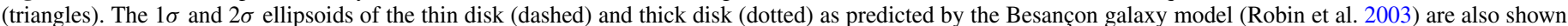
for comparison. The space velocity of LHS $2397 \mathrm{a}$ is consistent with other ultracool dwarfs, and we derive a $99.2 \%$ thin disk membership probability.

(A color version of this figure is available in the online journal.)

given object belonging to the thin and thick disks, we simply counted the nearest $10^{3}$ model objects in $(U, V, W)$-space and computed the fraction that belonged to each population. For LHS 2397 a, we found a membership probability of $99.2 \%$ for the thin disk and $0.8 \%$ for the thick disk. ${ }^{17}$

Finally, the fact that LHS 2397a is an active $\mathrm{H} \alpha$ flare star $\left(\log \left(L_{\mathrm{H} \alpha} / L_{\mathrm{bol}}\right)=-4.22\right.$; Gizis et al. 2000) could also potentially provide an age constraint, as the activity of $\mathrm{M}$ dwarfs changes with age. West et al. (2008) showed that the fraction of active $\mathrm{M}$ dwarfs as a function the vertical distance above the Galactic plane $(z)$ provides a constraint on the activity lifetime of $\mathrm{M}$ dwarfs, given a model of how thick disk heating pumps up $z$ over time. West et al. (2008) found that the activity lifetime increases monotonically with $\mathrm{M}$ dwarf spectral type, and the latest type for which they were able to determine a robust lifetime was M7 $\left(8.0_{-1.0}^{+0.5} \mathrm{Gyr}\right)$. This provides a weak constraint on the age of LHS 2397a, as its activity is therefore expected to last for at least $\gtrsim 8 \mathrm{Gyr}$.

In summary, the space motion of LHS 2397a indicates that it is (1) consistent with the field ultracool dwarf population, and (2) most likely $(99.2 \%)$ a member of the thin disk. These properties do not strongly constrain its age: the age of the ultracool field dwarf population is poorly constrained by current observations, and the thin disk is believed to have been forming stars continuously over the past $\sim 10$ Gyr. The activity of LHS 2397a is expected to be long lived, and so it also does not strongly constrain the age. Thus, the $1 \sigma$ age range of $1.0-10 \mathrm{Gyr}$ derived from evolutionary models for LHS 2397a (Section 4.1) is consistent with the broad range of ages allowed by its space motion and activity.

\footnotetext{
17 We also computed membership probabilities for all the ultracool dwarfs shown in Figure 9 and found only three systems with $>10 \%$ probability of belonging to the thick disk: ESO 207-61 (69\%), GJ 1001 (40\%), and G1 802 $(36 \%)$.
}

\section{TESTS OF MODELS}

Mass is the primary input parameter for evolutionary models of ultracool dwarfs. Thus, the strongest tests of these models are made possible by objects with a direct observational constraint on their mass. In the following analysis, we have used our derived total mass of LHS 2397aAB along with its other measured properties to test theoretical models. We drew the measured properties of LHS $2397 \mathrm{aAB}$ from appropriate random distributions, carefully accounting for the covariance between different quantities (e.g., $M_{\text {tot }}$ and $L_{\text {bol }}$ are correlated through the distance). We have chosen to use $L_{\text {bol }}$ rather than $T_{\text {eff }}$ as the basis of our model comparisons because values of $T_{\text {eff }}$ in the literature are invariably tied to either evolutionary or atmospheric theoretical models in some way due to the lack of direct radius measurements for brown dwarfs. By using $L_{\text {bol }}$, which only depends on direct measurements of SEDs and distances, we have avoided circular comparisons. This approach follows our previous work for 2MASS J1534-2952AB (Liu et al. 2008) and HD 130948BC (Dupuy et al. 2009).

In the following, we consider two independent sets of evolutionary models: the Tucson models (Burrows et al. 1997) and the Lyon DUSTY models (Chabrier et al. 2000), which are appropriate for both components of LHS $2397 \mathrm{aAB}$, as their photospheres are expected to be in the effective temperature range over which appreciable amounts of dust exist.

\subsection{Model-Inferred Age}

As described in detail by Liu et al. (2008) and Dupuy et al. (2009), the total mass of a binary along with its individual component luminosities can be used to estimate the age of the binary from evolutionary models. This age estimate can be surprisingly precise when both components are likely to be substellar since their luminosities depend very sensitively on age. For LHS 2397aAB, which only has one substellar component, we derive an age of $1.5_{-0.6}^{+4.1} \mathrm{Gyr}$ from the Tucson 


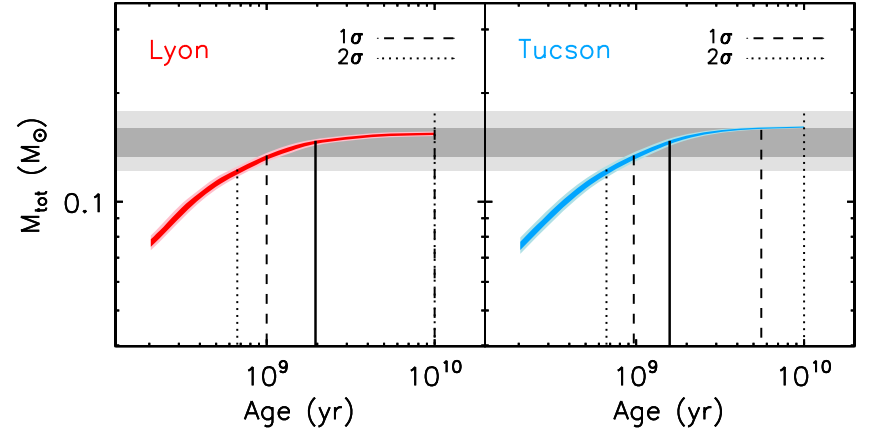

Figure 10. Total mass $\left(M_{\mathrm{tot}}\right)$ predicted by evolutionary models as a function of age, given the observational constraint of the luminosities of the two components of LHS $2397 \mathrm{aAB}$. The curved shaded regions show the $1 \sigma$ and $2 \sigma$ ranges in this model-derived mass. By applying the additional constraint of the measured total mass $\left(M_{\mathrm{tot}}\right)$, we used the models to determine the age of LHS 2397aAB (see Section 4.1). The horizontal gray bars show our $1 \sigma$ and $2 \sigma$ constraints on the total mass, and the resulting median, $1 \sigma$, and $2 \sigma$ model-inferred ages are shown by solid, dashed, and dotted lines, respectively. Model-inferred ages are truncated at $10 \mathrm{Gyr}$ (the oldest age included in both sets of models) - this happens at $1 \sigma$ for the Lyon models and $2 \sigma$ for the Tucson models. This is because the high-mass tail of the $M_{\text {tot }}$ distribution corresponds to both LHS 2397aA and LHS $2397 \mathrm{aB}$ being stars at the bottom of the main sequence, and evolutionary models do not reach faint enough luminosities for such high-mass objects by 10 Gyr.

(A color version of this figure is available in the online journal.)

models and an age of $1.8_{-0.8}^{+8.2}$ Gyr from the Lyon models (Figure 10).

Note that there is an upper limit of $10 \mathrm{Gyr}$ on the ages estimated from evolutionary models as this is the oldest age included in both sets of models. Our age estimates for LHS 2397aAB hit this limit at $1 \sigma$ for the Lyon models because the $1 \sigma$ upper limit on total mass $\left(0.160 M_{\odot}\right)$ corresponds to both LHS $2397 \mathrm{aA}$ and LHS $2397 \mathrm{aB}$ being stars at the bottom of the main sequence, and the Lyon models do not reach faint enough luminosities for such high-mass objects by $10 \mathrm{Gyr}$. This is also the case for the Tucson models, though the limit is reached beyond the $1 \sigma$ upper limit in the total mass. Using models that extend to older ages (e.g., the age of the universe) would not remedy this problem because LHS 2397aB's luminosity is much too low for a star even though the total mass formally allows it to be one (see Section 4.2).

\subsection{Individual Masses}

By measuring the relative orbit of LHS 2397aAB, we have determined its total mass to a precision of $10 \%$ (dominated by the error in the parallax). One way to estimate individual masses is to use evolutionary models in the same way they were used to infer the system age. By constraining the modelderived individual masses of LHS 2397aA and LHS 2397aB to add up to the observed total mass, while still matching their observed luminosities, the Tucson models give masses of $0.0839_{-0.0015}^{+0.0007} M_{\odot}$ and $0.061_{-0.011}^{+0.014} M_{\odot}$ for LHS $2397 \mathrm{aA}$ and LHS $2397 \mathrm{aB}$, respectively. The Lyon models give masses of $0.0848_{-0.0012}^{+0.0010} M_{\odot}$ and $0.060_{-0.012}^{+0.008} M_{\odot}$. We can also estimate the mass ratio $\left(q \equiv M_{\mathrm{B}} / M_{\mathrm{A}}\right)$ in this fashion, and the Tucson models give $q=0.73_{-0.12}^{+0.16}$, while the Lyon models give $q=$ $0.71_{-0.14}^{+0.09}$. This is among the lowest field ultracool binary mass ratios (e.g., see Burgasser et al. 2007) and is consistent with the original estimate of 0.76 by Freed et al. (2003) based on their photometry and estimated age range (2-12 Gyr).

We can also estimate the individual masses of LHS 2397aAB by subtracting the mass of the stellar primary from the total mass. There are only three stars of comparable spectral type and absolute magnitude to LHS 2397aA with dynamical mass estimates: LHS 1070B (M8.5, $M_{V}=19.24 \pm 0.07 \mathrm{mag}, M_{K}=$ $10.42 \pm 0.04$ mag; Leinert et al. 1994, 2000), LHS 1070C (M9, $M_{V}=19.62 \pm 0.08 \mathrm{mag}, M_{K}=10.76 \pm 0.04 \mathrm{mag}$; Leinert et al. 1994, 2000), and GJ 1245C $\left(M_{V}=18.55 \pm 0.05 \mathrm{mag}\right.$, $M_{K}=9.99 \pm 0.04 \mathrm{mag} ;$ Henry \& McCarthy 1993). The spectroscopically unclassified object GJ $1245 \mathrm{C}$ is the closest in absolute magnitude to LHS 2397aA and the only one of these with a direct dynamical mass measurement $(0.074 \pm$ $0.013 M_{\odot}$; Henry et al. 1999). LHS $1070 \mathrm{BC}$ only has a total mass determined from its relative orbit $\left(0.157 \pm 0.009 M_{\odot}\right.$; Seifahrt et al. 2008). If we simply assume that the mass of LHS 2397aA is the same as GJ $1245 \mathrm{C}$, then the resulting mass estimate for LHS $2397 \mathrm{aB}$ is $0.072 \pm 0.019 M_{\odot}$. This is somewhat larger than $(+0.6 \sigma)$ but consistent with the mass estimates from evolutionary models $\left(0.061_{-0.011}^{+0.014} M_{\odot}\right.$ and $\left.0.060_{-0.012}^{+0.008} M_{\odot}\right)$, which have somewhat smaller uncertainties. This approach is limited by the small number and low precision of dynamical masses for stars at the bottom of the main sequence, which will be improved by future mass measurements for late-M dwarf binaries.

The mass of LHS $2397 \mathrm{aB}$ is estimated to be substellar as it is below $0.070-0.092 M_{\odot}$, the range in the hydrogen-fusing minimum mass for plausible values of the metallicity and helium fraction (Burrows et al. 2001). This is not surprising since theoretical models predict that stars at the very bottom of the main sequence could not be as faint as LHS 2397aB. We can therefore estimate an upper limit on the total mass from the hydrogen-fusing minimum mass $\left(\approx 0.075 M_{\odot}\right.$ for solar metallicity) and the highest mass expected for LHS 2397aA. There are few mass measurements at the bottom of the main sequence, but we estimate that a conservative upper limit on the mass of LHS $2397 \mathrm{aA}$ is $0.095 M_{\odot}$. Gl $866 \mathrm{C}$ has a mass of $0.0930 \pm 0.0008 M_{\odot}$ and at $M_{V}=17.4 \pm 0.4 \mathrm{mag}$ is more than a magnitude brighter than LHS 2397aA; no objects with masses $>0.100 M_{\odot}$ come within 2 mag of LHS 2397aA's $M_{V}$. These upper limits on the masses of the individual components of LHS $2397 \mathrm{aAB}$ imply $M_{\mathrm{tot}}<0.170 M_{\odot}, 1.7 \sigma$ larger than our derived dynamical mass. Thus, we suggest that the high-mass tail of the $M_{\text {tot }}$ distribution, while formally allowed by the data, is practically excluded based on the properties of the individual components. $^{18}$

\subsection{Temperatures and Surface Gravities}

Without radius measurements for LHS 2397aA and LHS $2397 \mathrm{aB}$, we cannot directly determine their effective temperatures or surface gravities. ${ }^{19}$ However, we have used evolutionary models to estimate these properties in the same fashion as our model-derived age and individual masses.

The Lyon models give effective temperatures for LHS 2397aA and LHS $2397 \mathrm{aB}$ of $2470 \pm 30 \mathrm{~K}$ and $1430 \pm 40 \mathrm{~K}$, while the

\footnotetext{
18 If LHS 2397a were a higher order multiple, then its total mass would be more consistent with the high-mass tail of the $M_{\text {tot }}$ distribution. However, the fact that the luminosities and colors of both components agree well with single field dwarfs makes unresolved multiplicity unlikely.

19 Since LHS 2397aA is a chromospherically active star in a binary system, it may be possible to estimate its radius using the technique employed by Berger et al. (2009) who measured the rotation period of 2MASSW

J0746425+200032A from its chromospheric radio emission and combined this with its $v \sin (i)$ and orbital inclination $(i)$ to derive its radius. This method assumes that the orbital and rotation axes are aligned. With a $v \sin (i)$ of $20 \mathrm{~km}$ $\mathrm{s}^{-1}$ (Mohanty \& Basri 2003), LHS 2397aA is expected to have a rotation period of about 240 minutes.
} 
Tucson models give systematically hotter but formally consistent temperatures of $2580 \pm 30 \mathrm{~K}$ and $1450 \pm 40 \mathrm{~K}$. Because of the nearly flat mass-radius relation for such low-mass objects, it is essentially the uncertainties in the component luminosities that determine the precision in the model-derived effective temperatures for LHS 2397aA. Since brown dwarfs cool over time, the precision in LHS $2397 \mathrm{aB}$ 's effective temperature also depends somewhat on the uncertainty in the age, which in turn depends on the mass error. While our uncertainties were derived specifically for this system, approximate scaling relations derived by Liu et al. (2008) can provide simple estimates of the relative dependencies of the mass and luminosity errors on the uncertainty in $T_{\text {eff }}$.

The Lyon models give surface gravities for LHS 2397aA and LHS $2397 \mathrm{aB}$ of $\log (\mathrm{g})=5.307_{-0.008}^{+0.007}$ and $5.31_{-0.15}^{+0.09}(\mathrm{cgs})$, while the Tucson models give systematically higher gravities of $\log (g)=5.381_{-0.014}^{+0.009}$ and $5.35_{-0.13}^{+0.14}(\mathrm{cgs})$. Since the radius is essentially constant with age, the precision in model-inferred surface gravity is driven by the precision in the measured total mass. The difference between the two sets of modelinferred surface gravities, which are formally inconsistent for LHS $2397 \mathrm{aA}$, arises from small differences $(\lesssim 9 \%)$ in the modelpredicted radii (Table 5).

\subsubsection{Comparison to Field Dwarfs}

The effective temperatures we derive from evolutionary models for LHS 2397aA and LHS 2397aB can be compared to those which have been determined for other objects of similar spectral type. Such estimates in the literature utilize the nearly flat mass-radius relationship predicted by theoretical models for brown dwarfs, adopting either a typical age (e.g., 3 Gyr; Golimowski et al. 2004) or radius (e.g., $0.90 \pm 0.15 R_{\odot}$; Vrba et al. 2004).

For LHS 2397aA (M8 \pm 0.5 ), field M7-M9 dwarfs with $L_{\text {bol }}$ measurements have estimated effective temperatures ranging between 1850 and $2650 \mathrm{~K}$ (Leggett et al. 2001). For LHS 2397aB (L7 \pm 1 ), field L6-L8 dwarfs with $L_{\text {bol }}$ measurements have estimated effective temperatures ranging between 1300 and $1700 \mathrm{~K}$. Both of these broad ranges of effective temperature are consistent with our model-inferred effective temperatures. However, since both estimates are based on evolutionary models, this only means that field L6-L8 dwarfs from previous studies encompass objects of the same mass/age as LHS 2397aB.

Gautier et al. (2007) used their $24 \mu \mathrm{m}$ Spitzer/MIPS photometry to determine effective temperatures for nine M7.5M8.5 dwarfs (including LHS 2397a) using the nearly modelindependent infrared flux method. These temperatures range from 2400 to $2730 \mathrm{~K}$ (excluding LHS 2397a). They derived an effective temperature of $2380 \mathrm{~K}$ for LHS 2397a, but LHS 2397 aB likely affects this estimate since it is not a negligible source of flux at $24 \mu \mathrm{m}$ : the atmospheric models of Burrows et al. (2006) predict that it would contribute $0.16 \mathrm{mJy}$ of the measured $0.99 \mathrm{mJy}$ at $24 \mu \mathrm{m}$. This is likely why the $K_{S}-$ [24] color of LHS $2397 \mathrm{a}(1.09 \pm 0.10 \mathrm{mag})$ is redder than any other object of its spectral type (mean and rms of $0.80 \pm 0.08 \mathrm{mag}$ ), and this could explain why the temperature estimate for LHS 2397a is outside the range of the other eight M7.5-M8.5 dwarfs in the Gautier et al. (2007) sample. The infrared flux method temperature range of the other M7.5-M8.5 dwarfs (2400-2730 K) is in excellent agreement with the evolutionary model inferred effective temperatures for LHS 2397aA.
Table 4

Measured Properties of LHS 2397aAB

\begin{tabular}{|c|c|c|c|}
\hline Property $^{\mathrm{a}}$ & LHS 2397aA & LHS 2397aB & Ref. \\
\hline$M_{\text {tot }}\left(M_{\odot}\right)$ & \multicolumn{2}{|c|}{$0.146 \pm 0.014$} & 1 \\
\hline Semimajor axis (AU) & \multicolumn{2}{|c|}{$3.09 \pm 0.10$} & 1,2 \\
\hline$d(\mathrm{pc})$ & \multicolumn{2}{|c|}{$14.3 \pm 0.4$} & 2 \\
\hline Spectral Type & $\mathrm{M} 8 \pm 0.5$ & $\mathrm{~L} 7 \pm 1$ & 1,3 \\
\hline$J$ (mag) & $12.11 \pm 0.02$ & $15.23 \pm 0.08$ & 1,4 \\
\hline$H$ (mag) & $11.26 \pm 0.02$ & $14.22 \pm 0.05$ & 1,4 \\
\hline$K(\mathrm{mag})$ & $10.83 \pm 0.02$ & $13.64 \pm 0.04$ & 1,4 \\
\hline$L^{\prime}(\mathrm{mag})$ & $10.20 \pm 0.02$ & $12.12 \pm 0.05$ & 1,5 \\
\hline$J-H(\mathrm{mag})$ & $0.85 \pm 0.03$ & $1.01 \pm 0.10$ & 1,4 \\
\hline$H-K(\mathrm{mag})$ & $0.43 \pm 0.03$ & $0.58 \pm 0.07$ & 1,4 \\
\hline$J-K(\mathrm{mag})$ & $1.28 \pm 0.03$ & $1.59 \pm 0.09$ & 1,4 \\
\hline$K-L^{\prime}(\mathrm{mag})$ & $0.63 \pm 0.03$ & $1.51 \pm 0.07$ & $1,4,5$ \\
\hline$M_{V}(\mathrm{mag})$ & $18.80 \pm 0.07$ & $\ldots$ & 2 \\
\hline$M_{J}(\mathrm{mag})$ & $11.34 \pm 0.07$ & $14.46 \pm 0.10$ & $1,2,4$ \\
\hline$M_{H}(\mathrm{mag})$ & $10.49 \pm 0.07$ & $13.45 \pm 0.08$ & $1,2,4$ \\
\hline$M_{K}(\mathrm{mag})$ & $10.06 \pm 0.07$ & $12.87 \pm 0.08$ & $1,2,4$ \\
\hline$M_{L^{\prime}}(\mathrm{mag})$ & $9.43 \pm 0.07$ & $11.35 \pm 0.09$ & $1,2,5$ \\
\hline $\log \left(L_{\mathrm{bol}} / L_{\odot}\right)$ & $-3.42 \pm 0.03$ & $-4.52 \pm 0.03$ & 1 \\
\hline$\Delta \log \left(L_{\mathrm{bol}}\right)$ & 1.1 & 0.011 & 1 \\
\hline
\end{tabular}

Note. ${ }^{\text {a }}$ All near-infrared photometry on the MKO system.

References. (1) This work; (2) Monet et al. 1992; (3) Kirkpatrick et al. 1995; (4) Cutri et al. 2003; (5) Leggett et al. 2002.

\subsubsection{Comparison to Atmospheric Models}

Effective temperatures have also been determined for many field dwarfs through spectral synthesis modeling. By comparing these results to our evolutionary model-derived temperatures, we perform a consistency check between these two classes of models, which are essentially independent. Even though atmospheric models provide an important boundary condition for evolutionary models, very similar bulk properties (e.g., $L_{\text {bol }}$ and $\left.T_{\text {eff }}\right)$ are predicted by evolutionary models using widely varying boundary conditions (Chabrier \& Baraffe 2000; Saumon \& Marley 2008).

Using spectral synthesis modeling over a very narrow spectral range (2.297-2.310 $\mu \mathrm{m})$, Jones et al. (2005) found effective temperatures of $2900 \mathrm{~K}$ for the two M7-M9 dwarfs in their study. Using a broader spectral range (1.0-2.5 $\mu \mathrm{m})$, Leggett et al. (2001) found much cooler temperatures of $2100-2300 \mathrm{~K}$ for the five M7-M9 dwarfs in their study. The evolutionary model-inferred range of effective temperatures for LHS 2397aA $(2440-2610 \mathrm{~K})$ is inconsistent with both results from spectral synthesis modeling. Both authors cite the treatment of dust as the likely cause of discrepancies they found between the atmospheric models and their spectra.

Using spectral synthesis modeling over a very broad spectral range (0.95-14.5 $\mu \mathrm{m})$, Cushing et al. (2008) derived effective temperatures for several field $\mathrm{L}$ and $\mathrm{T}$ dwarfs, including two late-L dwarfs of comparable spectral type and $J H K$ color to LHS 2397aB. These two objects (2MASS J0825+2115, L7.5; DENIS J0255-4700, L8) were both found to have effective temperatures of $1400 \mathrm{~K}$, which is in perfect agreement with our model-derived temperature estimates for LHS 2397aB. This is somewhat surprising as previous studies of $\mathrm{L}$ and $\mathrm{T}$ dwarfs have found large $(100-200 \mathrm{~K})$ discrepancies between temperatures derived from atmospheric and evolutionary models (Liu et al. 2008; Dupuy et al. 2009). Figure 11 shows the atmospheric model predicted temperature for LHS 2397aB on the Hertzsprung-Russell (H-R) diagram with the evolutionary model tracks. It is interesting to note that both components of 
Table 5

Evolutionary Model-derived Properties of LHS 2397aAB

\begin{tabular}{|c|c|c|c|}
\hline Property & Median & $68.3 \%$ c.l. & $95.4 \%$ c.l. \\
\hline \multicolumn{4}{|c|}{ Tucson Models (Burrows et al. 1997) } \\
\hline \multicolumn{4}{|c|}{ System } \\
\hline Age $(G y r)^{a}$ & 1.5 & $-0.6,4.1$ & $-0.9,8.5$ \\
\hline$q\left(M_{\mathrm{B}} / M_{\mathrm{A}}\right)$ & 0.73 & $-0.12,0.16$ & $-0.19,0.18$ \\
\hline$\Delta T_{\text {eff }}(\mathrm{K})$ & 1130 & $-40,30$ & $-50,60$ \\
\hline \multicolumn{4}{|c|}{ Component A } \\
\hline$M_{\mathrm{A}}\left(M_{\odot}\right)$ & 0.0839 & $-0.0015,0.0007$ & $-0.005,0.001$ \\
\hline$T_{\text {eff,A }}(\mathrm{K})$ & 2580 & $-30,30$ & $-50,50$ \\
\hline $\log \left(g_{\mathrm{A}}\right)(\mathrm{cgs})$ & 5.381 & $-0.014,0.009$ & $-0.04,0.02$ \\
\hline$R_{\mathrm{A}}\left(R_{\odot}\right)$ & 0.0978 & $-0.0012,0.0012$ & $-0.002,0.003$ \\
\hline $\mathrm{Li}_{\mathrm{A}} / \mathrm{Li}_{0}$ & 0.00 & $-0.00,0.00$ & $-0.00,0.00$ \\
\hline \multicolumn{4}{|c|}{ Component B } \\
\hline$M_{\mathrm{B}}\left(M_{\odot}\right)$ & 0.061 & $-0.011,0.014$ & $-0.019,0.015$ \\
\hline$T_{\text {eff,B }}(\mathrm{K})$ & 1450 & $-40,40$ & $-80,70$ \\
\hline $\log \left(g_{\mathrm{B}}\right)(\mathrm{cgs})$ & 5.35 & $-0.13,0.14$ & $-0.25,0.15$ \\
\hline$R_{\mathrm{B}}\left(R_{\odot}\right)$ & 0.0868 & $-0.005,0.005$ & $-0.005,0.009$ \\
\hline $\mathrm{Li}_{\mathrm{B}} / \mathrm{Li}_{0}$ & 0.5 & $-0.5,0.5$ & $-0.5,0.5$ \\
\hline \multicolumn{4}{|c|}{ Lyon Models (DUSTY; Chabrier et al. 2000) } \\
\hline \multicolumn{4}{|c|}{ System } \\
\hline Age $(G y r)^{a}$ & 1.8 & $-0.8,8.2$ & $-1.1,8.2$ \\
\hline$q\left(M_{\mathrm{B}} / M_{\mathrm{A}}\right)$ & 0.71 & $-0.14,0.09$ & $-0.21,0.10$ \\
\hline$\Delta T_{\text {eff }}(\mathrm{K})$ & 1040 & $-40,50$ & $-50,80$ \\
\hline \multicolumn{4}{|c|}{ Component A } \\
\hline$M_{\mathrm{A}}\left(M_{\odot}\right)$ & 0.0848 & $-0.0012,0.0010$ & $-0.004,0.002$ \\
\hline$T_{\text {eff,A }}(\mathrm{K})$ & 2470 & $-30,30$ & $-50,60$ \\
\hline $\log \left(g_{\mathrm{A}}\right)(\mathrm{cgs})$ & 5.307 & $-0.008,0.007$ & $-0.03,0.01$ \\
\hline$R_{\mathrm{A}}\left(R_{\odot}\right)$ & 0.1073 & $-0.0010,0.0011$ & $-0.002,0.002$ \\
\hline $\mathrm{Li}_{\mathrm{A}} / \mathrm{Li}_{0}$ & 0.00 & $-0.00,0.00$ & $-0.00,0.00$ \\
\hline \multicolumn{4}{|c|}{ Component B } \\
\hline$M_{\mathrm{B}}\left(M_{\odot}\right)$ & 0.060 & $-0.012,0.008$ & $-0.020,0.009$ \\
\hline$T_{\text {eff,B }}(\mathrm{K})$ & 1430 & $-50,40$ & $-90,70$ \\
\hline $\log \left(g_{\mathrm{B}}\right)(\mathrm{cgs})$ & 5.31 & $-0.15,0.09$ & $-0.28,0.09$ \\
\hline$R_{\mathrm{B}}\left(R_{\odot}\right)$ & 0.0895 & $-0.0041,0.0064$ & $-0.004,0.012$ \\
\hline $\mathrm{Li}_{\mathrm{B}} / \mathrm{Li}_{0}$ & 0.0 & $-0.0,0.9$ & $-0.0,1.0$ \\
\hline
\end{tabular}

Note. ${ }^{a}$ Both sets of evolutionary models are only computed up to an age of $10 \mathrm{Gyr}$; therefore, this defines the upper limit on the model-derived ages.

the L4+L4 binary HD 130948BC were found to lie above the evolutionary model tracks (Dupuy et al. 2009), both components of the T5+T5.5 binary 2MASS J1534-2952AB were found to lie below the tracks (Liu et al. 2008), and LHS 2397aB with an intermediate spectral type of L7 falls exactly on the tracks. This suggests that whatever different systematic errors are harbored in each class of models may cancel out on the H-R diagram at this spectral type.

Cushing et al. (2008) also derived surface gravities for the two late-L dwarfs in their study by direct model fitting $(\log (g)=4.5-5.5)$ and by using their evolutionary sequences $(\log (g)=5.4-5.5)$. The latter values were determined from the atmospheric model $T_{\text {eff }}$ and the radius derived from the flux normalization constant $(R / d)^{2}$, which yields the radius if the distance is known. This is because atmospheric models predict the emergent flux in absolute units, which can be compared directly to flux-calibrated spectra. With independent estimates of both $R$ and $T_{\text {eff }}$ (from atmosphere fitting), any other property can be derived using evolutionary models. The surface gravities derived by Cushing et al. (2008) in this way for these two objects are higher by $0.4-1.9 \sigma$ than our evolutionary

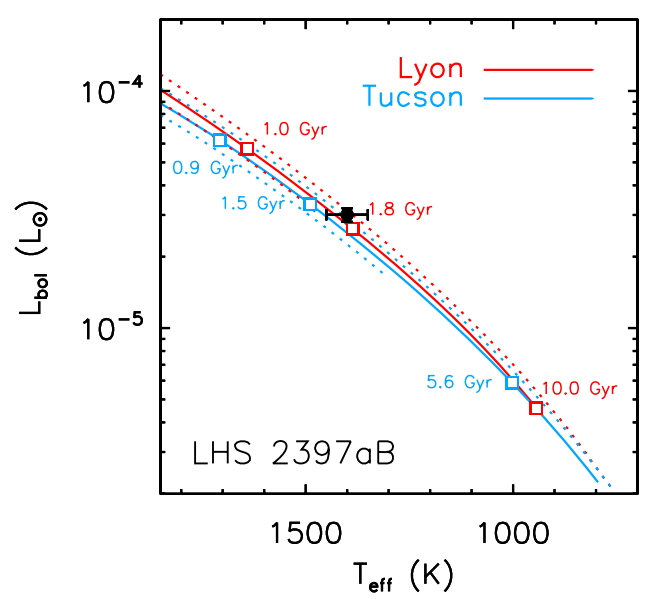

Figure 11. H-R diagram showing isomass lines from evolutionary models for the mass of LHS $2397 \mathrm{aB}$ with dotted lines encompassing the $1 \sigma$ uncertainty in the mass. The effective temperature of field L7 \pm 1 dwarfs as determined from spectral synthesis $(1400 \mathrm{~K})$ is shown as a filled circle with $50 \mathrm{~K}$ error bars, which correspond to the atmospheric model grid size. The open squares demarcate the median and $1 \sigma$ confidence limits on the evolutionary model-derived age of LHS 2397aAB, using the combined constraint of the total mass and individual luminosities (see Figure 10). The atmospheric model temperature is in very good agreement with the evolutionary models, unlike previous studies which have found 100-200 K discrepancies on the H-R diagram (Liu et al. 2008; Dupuy et al. 2009).

(A color version of this figure is available in the online journal.)

model inferred values for LHS $2397 \mathrm{aB}$ from Lyon and Tucson models $(\log (g)=5.31-5.35)$.

\subsection{Near-Infrared Colors}

The Lyon evolutionary models provide predictions of the fluxes of ultracool dwarfs in standard filter bandpasses as a function of model mass and age. ${ }^{20}$ We have derived the model-predicted near-infrared colors of both components of LHS $2397 \mathrm{aAB}$ in the same fashion as the individual masses, effective temperatures, and age (i.e., using the combined observational constraints of the total mass and individual luminosities). For LHS 2397aA, the Lyon DUSTY models predict a very narrow range of colors: $J-K=0.784 \pm 0.007 \mathrm{mag}$, $H-K=0.286 \pm 0.002 \mathrm{mag}$, and $J-H=0.498 \pm 0.006$ mag. None of these are consistent with the observed colors of LHS 2397aA: $J-K=1.28 \pm 0.03 \mathrm{mag}, H-K=0.43 \pm 0.03$ mag, and $J-H=0.85 \pm 0.03 \mathrm{mag}$. All of the observed colors are redder than predicted, with $J-K$ being the most discrepant (0.50 mag), followed by $J-H(0.35 \mathrm{mag})$ and $H-K(0.14 \mathrm{mag})$. Since the $J-K$ color of LHS $2397 \mathrm{aA}$ is consistent with other field dwarfs of spectral type M8 (1.14 \pm 0.10 mag; e.g., Faherty et al. 2009), the Lyon DUSTY models will generally provide inaccurate estimates of the fundamental properties of late-M dwarfs from their near-infrared colors. In fact, it would not be possible to estimate the mass of LHS 2397aA from the colormagnitude diagram as no model isochrone is consistent with the data.

We show the model-predicted $J H K L^{\prime}$ colors of LHS 2397aB compared to the observations and other field dwarfs in Figure 12. The colors of LHS $2397 \mathrm{aB}$ are fully consistent with field dwarfs of similar $K$-band absolute magnitude but are significantly bluer

\footnotetext{
20 The models give $J H K$ photometry on the CIT system, and we convert our measured MKO system colors to this system using the relations of (Stephens \& Leggett 2004). The model $L^{\prime}$-band photometry is given on the Johnson-Glass system, and no empirical relations between this and the MKO photometric system exist, so we neglect any differences.
} 


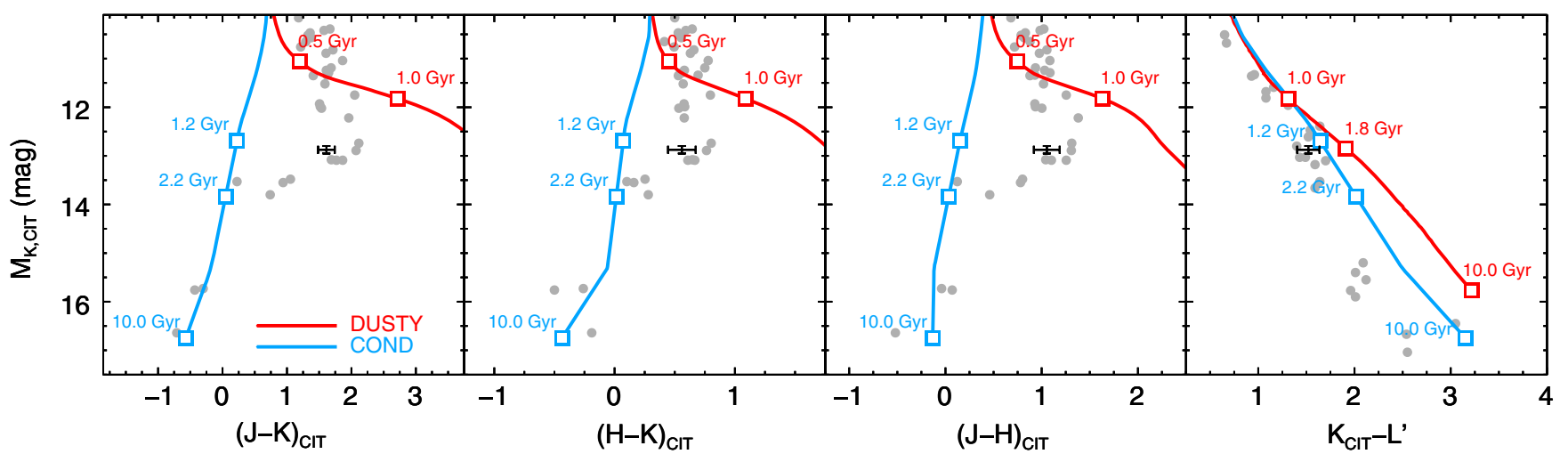

Figure 12. Color-magnitude diagrams showing the measured properties of LHS $2397 \mathrm{aB}$ compared to Lyon evolutionary tracks. The solid lines are isomass tracks from the DUSTY (Chabrier et al. 2000) and COND (Baraffe et al. 2003) models (JHK photometry on the CIT system and $L^{\prime}$-band photometry on the Johnson-Glass system). The open squares demarcate ages along the isomass lines, showing the median and $1 \sigma$ confidence limits on the model-inferred age of LHS $2397 \mathrm{aAB}$ if possible ( $0.5 \mathrm{Gyr}$ also shown for reference). Field dwarfs with parallax measurements better than $10 \%$ and $J H K$ colors better than 0.10 mag are shown as filled gray circles. LHS $2397 \mathrm{aB}$ has near-infrared colors typical of field dwarfs, but neither set of evolutionary models reproduces the observed colors of LHS 2397aB. This is not surprising since each model is a limiting case in the amount of dust in the photosphere (very dusty or no dust) and LHS 2397aB is expected to be intermediate given its estimated spectral type of $\mathrm{L} 7 \pm 1$. The $K-L^{\prime}$ colors predicted by models are by far the least discrepant with the data.

(A color version of this figure is available in the online journal.)

than the DUSTY models for any assumed age. This discrepancy with the models is not surprising as it is well known that the DUSTY models do not accurately reproduce the nearinfrared colors of late-L dwarfs. This is at least partly because DUSTY models represent the limiting case of very high dust content in the photosphere, while late-L dwarfs are actually in transition to dust-free atmospheres. For comparison, we also show the COND models (Baraffe et al. 2003), which represent the converse limiting case of a dust-free photosphere. As such, the COND models reproduce the colors of the bluest T dwarfs well, in which dust has sedimented below the photosphere, but they fail to reproduce the colors of LHS $2397 \mathrm{aB}$. For both DUSTY and COND models, the least discrepant color is $K-L^{\prime}$, which is only about $0.5 \mathrm{mag}$ bluer than predicted by DUSTY or COND, presumably because the effects of dust are less severe at longer wavelengths. The difficulty in modeling the colors of objects such as LHS $2397 \mathrm{aB}$ that are in transition from the L to T spectral class is well known, though models developed recently that attempt to account for the sedimentation of dust have enjoyed some success at reproducing observations (Burrows et al. 2006; Saumon \& Marley 2008).

\section{DISCUSSION}

\subsection{Effective Temperature of the L/T Transition}

Measurements of the fundamental properties of brown dwarfs undergoing the transition from $\mathrm{L}$ to $\mathrm{T}$ dwarfs are critical for understanding the physics governing dust in ultracool atmospheres. With a spectral type of L7 \pm 1 , LHS $2397 \mathrm{aB}$ is one such object for which our mass and luminosity measurements have enabled precise effective temperature and surface gravity estimates (Section 4.3). In fact, Liu et al. (2008) noted that "mass benchmarks" such as LHS $2397 \mathrm{aB}$ provide much more precise temperature estimates than "age benchmarks" (i.e., substellar companions to stars of known age) since the ages of field stars are notoriously difficult to estimate precisely (e.g., see Mamajek \& Hillenbrand 2008, and references therein). Metchev \& Hillenbrand (2006) suggested that the effective temperature of the $\mathrm{L} / \mathrm{T}$ transition varies with surface gravity (or equivalently, age), based on such age benchmark objects. Thus, it is interesting to compare the estimated temperature of LHS $2397 \mathrm{aB}$ to those of the few late-L and early- $T$ dwarf companions with age estimates:
Gl 584C (L8; Kirkpatrick et al. 2001), HD 203030B (L7.5; Metchev \& Hillenbrand 2006), and HN Peg B (T2.5; Luhman et al. 2007). ${ }^{21}$

For Gl 584C, Golimowski et al. (2004) derived an effective temperature range of $1300-1400 \mathrm{~K}$ from evolutionary models, using its measured luminosity $\left(\log L_{\mathrm{bol}} / L_{\odot}=-4.58 \pm 0.04\right)$ and estimated age range (1-2.5 Gyr). This is consistent with our temperature estimate for LHS $2397 \mathrm{aB}$ (1380-1470 K from Lyon models; $1410-1490 \mathrm{~K}$ from Tucson models). In a similar fashion, Metchev \& Hillenbrand (2006) and Luhman et al. (2007) estimated the effective temperature ranges for two companions to young stars: $1090-1280 \mathrm{~K}$ for HD 203030B (130-400 Myr) and 1060-1200 K for HN Peg B (100-500 Myr). Compared to Gl $584 \mathrm{C}$ and LHS $2397 \mathrm{aB}$, the temperatures of these young companions are much cooler. As discussed by Metchev \& Hillenbrand (2006), field dwarfs of similar spectral type (L7-L9) with measured luminosities also have higher estimated temperatures (mean and rms of $1460 \pm 100 \mathrm{~K}$; from Vrba et al. 2004), though these estimates depend strongly on the estimated age of the field population, which is not wellconstrained observationally.

Metchev \& Hillenbrand (2006) suggested two possible explanations for the observed differences in effective temperatures: (1) the age of the field population has been overestimated, and (2) the effective temperature of the $\mathrm{L} / \mathrm{T}$ transition depends on surface gravity. If field dwarfs are younger than estimated, their radii will be larger and thus lower effective temperatures are required to match their measured luminosities. Support for this possibility has come from Liu et al. (2008), who suggested that the age of field dwarfs may be overestimated by as much as a factor of $6 \pm 3$ (implying an age range of 0.3-1.0 Gyr for field objects) based on a $\approx 100 \mathrm{~K}$ discrepancy between the field population and their dynamical mass-based temperature estimates for the components of 2MASS J1534-2952AB (T5+T5.5). Such an overestimate would impact effective temperature estimates at all spectral types, not just the $\mathrm{L} / \mathrm{T}$ transition. Metchev \& Hillenbrand (2006) preferred the explanation that the L/T transition is gravity dependent, so that objects of different radii/

\footnotetext{
${ }^{21}$ Gl 337CD (L8+T; Wilson et al. 2001; Burgasser et al. 2005) is another such companion; however, it is a tight binary with poor constraints on its resolved properties. Thus, we exclude it from our discussion.
} 
ages would undergo this evolutionary phase at different effective temperatures. Our effective temperature determination for LHS $2397 \mathrm{aB}$ supports this idea since LHS $2397 \mathrm{aB}$ belongs to the field population (Section 3.4) and its temperature is inconsistent by $100-400 \mathrm{~K}$ with the young late-L companions HD 203030B and HN Peg B.

\subsection{Direct Measurement of the Mass Ratio}

Although the total dynamical mass we have measured enables strong tests of theoretical models, these tests would be even more potent with the direct measurement of the individual component masses. In principle, this can be done using radial velocity or astrometric monitoring, both of which provide a measurement of the binary's mass ratio. The radial velocity semi-amplitude of LHS $2397 \mathrm{aA}$ is $1.9 \mathrm{~km} \mathrm{~s}^{-1}$ for the mass ratio of 0.7 derived from evolutionary models (Section 4.2). Thus, a radial velocity precision of $0.1 \mathrm{~km} \mathrm{~s}^{-1}$ would be needed to achieve a mass ratio measurement precise to $10 \%$ (i.e., comparable to the precision in the total mass). As discussed in Section 3.1, Basri \& Reiners (2006) measured radial velocities for LHS 2397aA at two epochs separated by almost exactly half an orbital period, but with a precision of $1.6 \mathrm{~km} \mathrm{~s}^{-1}$ these data are not sufficient to determine the mass ratio.

To determine the individual masses from astrometric monitoring, the orbit of LHS 2397aA about the system's barycenter must be measured. The size of this orbit is 89 mas for a mass ratio of 0.7 . Therefore, an astrometric precision of 5 mas in the optical, where LHS $2397 \mathrm{aB}$ is essentially invisible, is all that would be needed to measure the mass ratio to $\approx 10 \%$ $\left(\sigma_{q} / q=(1+q) \sigma_{a_{1}} / a_{1}\right)$. Such a measurement is quite feasible, though it requires long-term observations given the orbital period of 14.2 years.

\subsection{Lithium Depletion}

The presence or absence of lithium has long been used as a tracer of the internal structure of objects, providing constraints on mass and/or age, because it is only destroyed by fusion at temperatures above $\approx 2.5 \times 10^{6} \mathrm{~K}$. There is a mass limit below which lithium is never destroyed in the fully convective interiors of very low mass stars, and this occurs at $\approx 0.06 M_{\odot}$ (Chabrier et al. 1996). The estimated mass of LHS $2397 \mathrm{aB}$ is extremely close to this limit, so $1 \sigma$ confidence limits on its model-derived lithium abundance given in Table 5 range from zero (fully depleted) to unity (no depletion). There are currently no objects with direct mass estimates to provide observational constraints on the lithium depletion boundary for brown dwarfs. Thus, observations of lithium absorption at $6708 \AA$ for LHS $2397 \mathrm{aB}$ would provide a strong test of theoretical models of brown dwarf interiors. In addition, such observations would provide an independent constraint on the mass and age of LHS 2397aB that would enable a test of model-predicted luminosity evolution. Lithium observations are only possible with a repaired Space Telescope Imaging Spectrograph (STIS) onboard HST, and the next few years are an ideal time for such a measurement as the orbital separation is currently approaching its maximum (Figure 4).

\section{CONCLUSIONS}

We have determined the orbit of the M8+L7 binary LHS 2397aAB using relative astrometry spanning 11.8 years of its 14.2 year orbit. The astrometry and corresponding errors used to derive this orbit were thoroughly examined through
Monte Carlo simulations. The resulting best-fit orbit has a reduced $\chi^{2}$ of 1.04 and a total mass of $0.146_{-0.013}^{+0.015} M_{\odot}$. The error in the dynamical mass $(10 \%)$ is dominated by the $3.0 \%$ error in the parallax, which translates into a $9.0 \%$ error in the mass. The total mass is consistent with the primary component being a star and the companion being a brown dwarf, as expected from their spectral types. The combined observational constraints of the total mass and individual luminosities break the mass-ageluminosity degeneracy for the substellar companion, enabling specific predictions of its properties from evolutionary models. Tucson models (Burrows et al. 1997) predict an age for the system of $1.5_{-0.6}^{+4.1} \mathrm{Gyr}$, and the Lyon (DUSTY; Chabrier et al. 2000) model-derived age of $1.8_{-0.8}^{+8.2} \mathrm{Gyr}$ is consistent. These ages are consistent with the (weak) observational constraints on the system: LHS 2397a's space motion implies it is a member of the thin disk (1-10 Gyr; e.g., Wood \& Oswalt 1998), and although it is chromospherically active, its expected activity lifetime is $\gtrsim 8$ Gyr (West et al. 2008).

LHS $2397 \mathrm{aB}(\mathrm{L} 7 \pm 1)$ is now the first mass benchmark at the transition from $\mathrm{L}$ to $\mathrm{T}$ spectral types. This enables a precise estimate of the effective temperature of this phase of substellar evolution from theoretical models, which give $1450 \pm 40 \mathrm{~K}$ (Tucson) and $1430 \pm 40 \mathrm{~K}$ (Lyon). This is $\approx 200 \mathrm{~K}$ higher than temperature estimates for late-L companions to young stars: HD 203030B (130-400 Myr; Metchev \& Hillenbrand 2006) and HN Peg B (100-500 Myr; Luhman et al. 2007). However, the effective temperature of LHS $2397 \mathrm{aB}$ is consistent with the 1300-1400 K range estimated for the older lateL companion Gl 584C (1-2.5 Gyr; Kirkpatrick et al. 2001). This supports the idea originally proposed by Metchev \& Hillenbrand (2006) that the L/T transition can occur over a wide range of effective temperatures due to a surface gravity dependence on the process. In addition, the temperature for LHS $2397 \mathrm{aB}$ is consistent with estimates for field late-L dwarfs $(1440 \pm 100 \mathrm{~K})$, and thus an overestimate of the age of the field population (or underestimate of radii) is not required to explain the range of temperatures observed at the $\mathrm{L} / \mathrm{T}$ transition, as previously suggested (Metchev \& Hillenbrand 2006; Liu et al. 2008).

We have determined the spectral type of LHS $2397 \mathrm{aB}$ from its $J H K L^{\prime}$ absolute magnitudes, using a novel technique that enables a quantitative assessment of the spectral type uncertainty by accounting for both measurement errors and the rms scatter in the empirical relations. Comparing to other objects of similar spectral type and color for which Cushing et al. (2008) have conducted spectral synthesis analysis allows us to test atmospheric models for consistency with evolutionary models. The Cushing et al. (2008) study included two late-L dwarfs, and their derived effective temperatures were both $1400 \mathrm{~K}$, which is consistent with the effective temperature of LHS $2397 \mathrm{aB}$ we derived. This is somewhat surprising as similar consistency tests by previous studies of binary brown dwarfs have found large (100-200 K) discrepancies between effective temperatures derived from atmospheric and evolutionary models. However, it is interesting to note that LHS $2397 \mathrm{aB}$ has a spectral type intermediate between HD 130948BC for which the effective temperatures are underestimated by atmospheric models (L4+L4; Dupuy et al. 2009) and 2MASS J1534-2952AB for which the temperatures are overestimated (T5+T5.5; Liu et al. 2008). This suggests that LHS $2397 \mathrm{aB}$ may be at a temperature where atmospheric and evolutionary models are in agreement because the significant systematic errors in the models cancel out.

Because of its extreme contrast ratio ( $\gtrsim 100: 1$ in the optical), LHS $2397 \mathrm{aAB}$ is one of the few ultracool binaries whose mass 
ratio can be readily measured through astrometric monitoring. Given the estimated mass ratio of 0.7 , the barycentric orbit of LHS $2397 \mathrm{aA}$ is 89 mas, which is easily measurable with current instrumentation. It is unlikely that LHS 2397aA's 14 year orbital motion significantly impacted the original optical parallax measurement, except by introducing additional scatter in the measurements. Thus, new astrometric monitoring observations should improve the parallax precision, which is essential for further testing of models. The total mass is currently constrained to a precision of $10 \%$, dominated by the error in the parallax, and at this precision the secondary component is formally allowed (at $1 \sigma-2 \sigma$ ) to be a star at the bottom of the main sequence. A more precise mass measurement for this system would enable much stronger constraints on all model-predicted properties, including the amount of lithium depletion in LHS $2397 \mathrm{aB}$. Its model-derived individual mass $\left(\approx 0.06 \pm 0.01 M_{\odot}\right)$ is very close to the theoretical mass limit for lithium burning, which has never been tested with direct mass measurements. A lithium measurement for a brown dwarf of known mass would enable a unique constraint on substellar models as lithium depletion proceeds rapidly and independently of luminosity evolution. Such a resolved measurement of the lithium doublet at $6708 \AA$ for LHS $2397 \mathrm{aB}$ is only possible with $H S T /$ STIS.

Mass benchmarks enable some of the strongest tests of theoretical models by providing measurements of one of the most fundamental physical parameters. We have determined the mass of an object in the L/T transition for the first time-a key step in understanding this complex phase of substellar evolution. Our ongoing Keck LGS AO orbital monitoring of L/T binaries, in which the spectral type of one component is late- $\mathrm{L}$ and the other is early-T, will soon yield dynamical masses for a variety of $\mathrm{L} / \mathrm{T}$ transition objects. These will provide mass benchmarks in the $\mathrm{L} / \mathrm{T}$ transition for a broad range of surface gravities, enabling even stronger tests of the evolution of ultracool atmospheres.

We gratefully acknowledge the Keck AO team for their exceptional efforts in bringing the $\mathrm{AO}$ system to fruition. It is a pleasure to thank Antonin Bouchez, David LeMignant, Marcos van Dam, Randy Campbell, Al Conrad, Jim Lyke, Hien Tran, Jason McIlroy, and Gary Punawai, and the Keck Observatory staff for assistance with the observations. We are very thankful for the contribution of Peter Tuthill in establishing aperture masking at Keck. We are grateful to Brian Cameron for making available his NIRC2 distortion solution, Céline Reylé for customized Besançon Galaxy models, and Adam Burrows and Isabelle Baraffe for providing finely gridded evolutionary models. We have benefited from discussions with Michael Cushing about theoretical models and Thierry Forveille about space motions and orbit fitting using ORBIT. We are indebted to Katelyn Allers for assistance in obtaining and reducing IRTF/SpeX data. We also acknowledge the referee's helpful comments on the organization of this paper. Our research has employed the 2MASS data products; NASA's Astrophysical Data System; the SIMBAD database operated at CDS, Strasbourg, France; the SpeX Prism Spectral Libraries, maintained by Adam Burgasser at http://www.browndwarfs.org/spexprism; and the M, L, and $\mathrm{T}$ dwarf compendium housed at DwarfArchives.org and maintained by Chris Gelino, Davy Kirkpatrick, and Adam Burgasser (Kirkpatrick 2003; Gelino et al. 2004). T.J.D. and M.C.L. acknowledge support for this work from NSF grant AST-0507833, and M.C.L. acknowledges support from an Alfred P. Sloan Research Fellowship. Finally, the authors wish to recognize and acknowledge the very significant cultural role and reverence that the summit of Mauna Kea has always had within the indigenous Hawaiian community. We are most fortunate to have the opportunity to conduct observations from this mountain.

Facilities: Keck II Telescope (LGS AO, NIRC2), HST (WFPC2), VLT (NACO), Gemini-North Telescope (Hokupa'a), IRTF (SpeX).

\section{REFERENCES}

Bailer-Jones, C. A. L. 2004, A\&A, 419, 703

Baraffe, I., Chabrier, G., Barman, T. S., Allard, F., \& Hauschildt, P. H. 2003, A\&A, 402, 701

Barman, T. S. 2008, ApJ, 676, L61

Basri, G., \& Reiners, A. 2006, AJ, 132, 663

Bate, M. R. 2009, MNRAS, 392, 590

Berger, E., et al. 2009, ApJ, 695, 310

Bremaud, P. 1999, Markov Chains: Gibbs Fields, Monte Carlo Simulation, and Queues (New York: Springer)

Burgasser, A. J., Kirkpatrick, J. D., \& Lowrance, P. J. 2005, AJ, 129, 2849

Burgasser, A. J., Liu, M. C., Ireland, M. J., Cruz, K. L., \& Dupuy, T. J. 2008, ApJ, 681,579

Burgasser, A. J., \& McElwain, M. W. 2006, AJ, 131, 1007

Burgasser, A. J., McElwain, M. W., Kirkpatrick, J. D., Cruz, K. L., Tinney, C. G., \& Reid, I. N. 2004, AJ, 127, 2856

Burgasser, A. J., Reid, I. N., Siegler, N., Close, L., Allen, P., Lowrance, P., \& Gizis, J. 2007, in Protostars and Planets V, ed. B. Reipurth, D. Jewitt, \& K. Keil (Tucson, AZ: Univ. Arizona Press), 427

Burrows, A., Hubbard, W. B., Lunine, J. I., \& Liebert, J. 2001, Rev. Mod. Phys., 73,719

Burrows, A., Sudarsky, D., \& Hubeny, I. 2006, ApJ, 640, 1063

Burrows, A., et al. 1997, ApJ, 491, 856

Chabrier, G., \& Baraffe, I. 2000, ARA\&A, 38, 337

Chabrier, G., Baraffe, I., Allard, F., \& Hauschildt, P. 2000, ApJ, 542, 464

Chabrier, G., Baraffe, I., \& Plez, B. 1996, ApJ, 459, L91

Cushing, M. C., Vacca, W. D., \& Rayner, J. T. 2004, PASP, 116, 362

Cushing, M. C., et al. 2008, ApJ, 678, 1372

Cutri, R. M., et al. 2003, 2MASS All Sky Catalog of point sources (The IRSA 2MASS All-Sky Point Source Catalog NASA/IPAC Infrared Science Archive. http://irsa.ipac.caltech.edu/applications/Gator/)

Dahn, C. C., et al. 2002, AJ, 124, 1170

Dupuy, T. J., Liu, M. C., \& Ireland, M. J. 2009, ApJ, 692, 729

Duquennoy, A., \& Mayor, M. 1991, A\&A, 248, 485

Faherty, J. K., Burgasser, A. J., Cruz, K. L., Shara, M. M., Walter, F. M., \& Gelino, C. R. 2009, AJ, 137, 1

Fischer, D. A., \& Marcy, G. W. 1992, ApJ, 396, 178

Fisher, R. T. 2004, ApJ, 600, 769

Forveille, T., et al. 1999, A\&A, 351, 619

Freed, M., Close, L. M., \& Siegler, N. 2003, ApJ, 584, 453

Gautier, T. N., III, et al. 2007, ApJ, 667, 527

Gelino, C. R., Kirkpatrick, J. D., \& Burgasser, A. J. 2004, BAAS, 36, 1354

Ghez, A. M., et al. 2008, ApJ, 689, 1044

Gizis, J. E., Monet, D. G., Reid, I. N., Kirkpatrick, J. D., Liebert, J., \& Williams, R. J. 2000, AJ, 120, 1085

Golimowski, D. A., et al. 2004, AJ, 127, 3516

Henry, T. J., Franz, O. G., Wasserman, L. H., Benedict, G. F., Shelus, P. J., Ianna, P. A., Kirkpatrick, J. D., \& McCarthy, D. W., Jr. 1999, ApJ, 512, 864

Henry, T. J., Jao, W.-C., Subasavage, J. P., Beaulieu, T. D., Ianna, P. A., Costa, E., \& Méndez, R. A. 2006, AJ, 132, 2360

Henry, T. J., \& Kirkpatrick, J. D. 1990, ApJ, 354, L29

Henry, T. J., \& McCarthy, D. W., Jr. 1993, AJ, 106, 773

Ibukiyama, A., \& Arimoto, N. 2002, A\&A, 394, 927

Ireland, M. J., \& Kraus, A. L. 2008, ApJ, 678, L59

Ireland, M. J., Kraus, A., Martinache, F., Lloyd, J. P., \& Tuthill, P. G. 2008, ApJ, 678,463

Jones, H. R. A., Pavlenko, Y., Viti, S., Barber, R. J., Yakovina, L. A., Pinfield, D., \& Tennyson, J. 2005, MNRAS, 358, 105

Jones, H. R. A., \& Tsuji, T. 1997, ApJ, 480, L39

Kirkpatrick, J. D. 2003, in Proc. IAU Symp. 211, Brown Dwarfs, ed. E. Martin (Dordrecht: Kluwer), 189

Kirkpatrick, J. D., Dahn, C. C., Monet, D. G., Reid, I. N., Gizis, J. E., Liebert, J., \& Burgasser, A. J. 2001, AJ, 121, 3235

Kirkpatrick, J. D., Henry, T. J., \& McCarthy, D. W., Jr. 1991, ApJS, 77, 417

Kirkpatrick, J. D., Henry, T. J., \& Simons, D. A. 1995, AJ, 109, 797

Kraus, A. L., Ireland, M. J., Martinache, F., \& Lloyd, J. P. 2008, ApJ, 679, 762 
Krist, J. 1995, in ASP Conf. Ser. 77, Astronomical Data Analysis Software and Systems IV, ed. R. A. Shaw, H. E. Payne, \& J. J. E. Hayes (San Francisco, CA: ASP), 349

Leggett, S. K., Allard, F., Geballe, T. R., Hauschildt, P. H., \& Schweitzer, A. 2001, ApJ, 548, 908

Leggett, S. K., et al. 2002, ApJ, 564, 452

Leinert, C., Allard, F., Richichi, A., \& Hauschildt, P. H. 2000, A\&A, 353, 691

Leinert, C., Weitzel, N., Richichi, A., Eckart, A., \& Tacconi-Garman, L. E. 1994, A\&A, 291, L47

Lenzen, R., et al. 2003, in SPIE Conf. 4841, ed. M. Iye \& A. F. M Moorwood (Bellingham, WA: SPIE), 944

Liebert, J., \& Gizis, J. E. 2006, PASP, 118, 659

Liu, M. C., Dupuy, T. J., \& Ireland, M. J. 2008, ApJ, 689, 436

Liu, M. C., Leggett, S. K., Golimowski, D. A., Chiu, K., Fan, X., Geballe, T. R., Schneider, D. P., \& Brinkmann, J. 2006, ApJ, 647, 1393

Lohmann, A. W., Weigelt, G., \& Wirnitzer, B. 1983, Appl. Opt., 22, 4028

Luhman, K. L., et al. 2007, ApJ, 654, 570

Luyten, W. J. 1979, LHS Catalogue: A Catalogue of Stars with Proper Motions Exceeding 0.'5 Annually (2nd ed.; Minneapolis, MN: Univ. Minnesota)

Mamajek, E. E., \& Hillenbrand, L. A. 2008, ApJ, 687, 1264

Marois, C., Macintosh, B., Barman, T., Zuckerman, B., Song, I., Patience, J., Lafrenière, D., \& Doyon, R. 2008, Science, 322, 1348

Martinache, F., Lloyd, J. P., Ireland, M. J., Yamada, R. S., \& Tuthill, P. G. 2007, ApJ, 661, 496

Metchev, S. A., \& Hillenbrand, L. A. 2006, ApJ, 651, 1166

Mohanty, S., \& Basri, G. 2003, ApJ, 583, 451

Monet, D. G., Dahn, C. C., Vrba, F. J., Harris, H. C., Pier, J. R., Luginbuhl, C. B., \& Ables, H. D. 1992, AJ, 103, 638

Monet, D. G., et al. 2003, AJ, 125, 984

Rayner, J. T., Toomey, D. W., Onaka, P. M., Denault, A. J., Stahlberger, W. E., Watanabe, D. Y., \& Wang, S. 1998, in Proc. SPIE 3354, Infrared Astronomical Instrumentation, ed. A. M. Fowler (Bellingham, WA: SPIE), 468
Robin, A. C., Reylé, C., Derrière, S., \& Picaud, S. 2003, A\&A, 409, 523

Rousset, G., et al. 2003, in SPIE Conf. 4839, Adaptive Optical System Technologies II, ed. P. L. Wizinowich \& D. Bonaccini (Bellingham, WA: SPIE), 140

Saumon, D., \& Marley, M. S. 2008, ApJ, 689, 1327

Seifahrt, A., Röll, T., Neuhäuser, R., Reiners, A., Kerber, F., Käufl, H. U., Siebenmorgen, R., \& Smette, A. 2008, A\&A, 484, 429

Simons, D. A., \& Tokunaga, A. 2002, PASP, 114, 169

Stephens, D. C., \& Leggett, S. K. 2004, PASP, 116, 9

Tegmark, M., et al. 2004, Phys. Rev. D, 69, 103501

Tinney, C. G. 1996, MNRAS, 281, 644

Tinney, C. G., Burgasser, A. J., \& Kirkpatrick, J. D. 2003, AJ, 126, 975

Tinney, C. G., \& Reid, I. N. 1998, MNRAS, 301, 1031

Tinney, C. G., Reid, I. N., Gizis, J., \& Mould, J. R. 1995, AJ, 110, 3014

Tokunaga, A. T., Simons, D. A., \& Vacca, W. D. 2002, PASP, 114, 180

Tsuji, T., Ohnaka, K., Aoki, W., \& Nakajima, T. 1996, A\&A, 308, L29

Tuthill, P., et al. 2006, in SPIE Conf. Ser. 6272, Advances in Adaptive Optics II, ed. B. L. Ellerbroek \& D. Bonaccini Calia (Bellingham, WA: SPIE), 62723A

Vacca, W. D., Cushing, M. C., \& Rayner, J. T. 2003, PASP, 115, 389

van Dam, M. A., et al. 2006, PASP, 118, 310

Vrba, F. J., et al. 2004, AJ, 127, 2948

West, A. A., Hawley, S. L., Bochanski, J. J., Covey, K. R., Reid, I. N., Dhital S., Hilton, E. J., \& Masuda, M. 2008, AJ, 135, 785

Wilson, J. C., Kirkpatrick, J. D., Gizis, J. E., Skrutskie, M. F., Monet, D. G., \& Houck, J. R. 2001, AJ, 122, 1989

Wizinowich, P. L., et al. 2006, PASP, 118, 297

Wood, M. A., \& Oswalt, T. D. 1998, ApJ, 497, 870

Zapatero Osorio, M. R., Lane, B. F., Pavlenko, Y., Martín, E. L., Britton, M., \& Kulkarni, S. R. 2004, ApJ, 615, 958

Zapatero Osorio, M. R., Martín, E. L., Béjar, V. J. S., Bouy, H., Deshpande, R., \& Wainscoat, R. J. 2007, ApJ, 666, 1205 